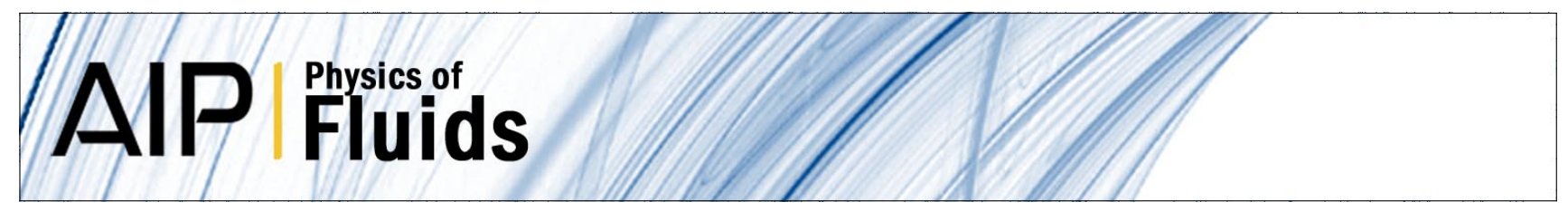

\title{
Experimental manipulation of wall turbulence: A systems approach
}

B. J. McKeon, A. S. Sharma, and I. Jacobi

Citation: Phys. Fluids 25, 031301 (2013); doi: 10.1063/1.4793444

View online: http://dx.doi.org/10.1063/1.4793444

View Table of Contents: http://pof.aip.org/resource/1/PHFLE6/v25/i3

Published by the American Institute of Physics.

\section{Additional information on Phys. Fluids}

Journal Homepage: http://pof.aip.org/

Journal Information: http://pof.aip.org/about/about_the_journal

Top downloads: http://pof.aip.org/features/most_downloaded

Information for Authors: http://pof.aip.org/authors

\section{ADVERTISEMENT}

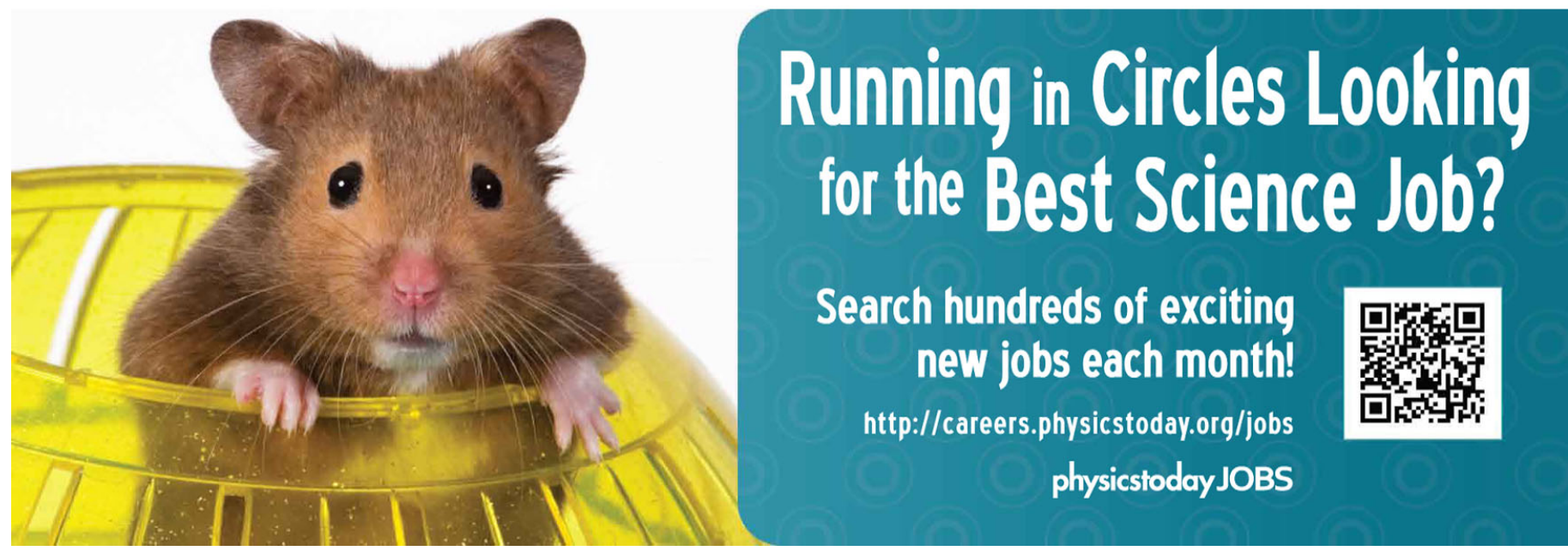




\title{
Experimental manipulation of wall turbulence: A systems approach ${ }^{\text {a) }}$
}

\author{
B. J. McKeon, ${ }^{1}$ A. S. Sharma, ${ }^{2}$ and I. Jacobi ${ }^{1}$ \\ ${ }^{1}$ Graduate Aerospace Laboratories, California Institute of Technology, Pasadena, \\ California 91125, USA \\ ${ }^{2}$ Automatic Control and Systems Engineering, University of Sheffield, Sheffield S1 3JD, \\ United Kingdom
}

(Received 22 August 2012; accepted 17 January 2013; published online 19 March 2013)

\begin{abstract}
We review recent progress, based on the approach introduced by McKeon and Sharma [J. Fluid Mech. 658, 336-382 (2010)], in understanding and controlling wall turbulence. The origins of this analysis partly lie in nonlinear robust control theory, but a differentiating feature is the connection with, and prediction of, state-of-the-art understanding of velocity statistics and coherent structures observed in real, high Reynolds number flows. A key component of this line of work is an experimental demonstration of the excitation of velocity response modes predicted by the theory using non-ideal, but practical, actuation at the wall. Limitations of the approach and promising directions for future development are outlined. ( 2013 American Institute of Physics. [http://dx.doi.org/10.1063/1.4793444]
\end{abstract}

\section{INTRODUCTION}

The potential impact of control of turbulence near walls, particularly in the context of the energy expenditure and emissions associated with overcoming turbulent skin friction, is well known. Aviation alone is known to consume approximately $13 \%$ of all transport-related energy requirements, ${ }^{2}$ of which up to $50 \%$ is associated with turbulent drag on commercial airliners. ${ }^{3,4} \mathrm{Kim}^{5}$ quantified the savings arising from the reduced fuel consumption associated with a relatively modest $30 \%$ reduction in skin friction achieved by employing future flow control technologies on ocean-going ships as $0.7 \times 10^{9}$ barrels of oil, or $\$ 70$ billion annually (updated to current prices of approximately $\$ 100$ per barrel). In terms of emissions, which would be similarly affected by reductions in fuel burn, a single long distance flight of a Boeing 747 is known to generate in excess of 400 tons of $\mathrm{CO}_{2}$. Alonso $\mathrm{et} \mathrm{al.}^{2}$ estimate that this is all the more potent as a greenhouse gas (by a factor of 2-4) because it is released at altitude.

At the level of fundamental understanding, the ability to effect the practical control required to obtain this reduction in applications such as commercial air vehicles would imply a significant advance in understanding of the dynamics and scaling of turbulence, together with a step away from the canonical flows that have been the primary focus of academic study for several decades. Clauser ${ }^{6}$ framed such an advance in the context of considering wall turbulence as a "black box," the contents of which can be explored by investigating its response to external stimuli. Where the response is unknown, the black-box idea approaches turbulence as a problem in nonlinear system identification. In contrast, the current approach considers the problem of response to endogenous stimulus, as determined by the Navier-Stokes equations. Since the equations are known, this is a white-box approach, to use the terminology of control engineering. With careful design of the form of the input, the linear and nonlinear mechanisms of energy transfer, or the pathways for energy transfer in physical and spectral space, can be identified.

\footnotetext{
a) This paper is based on an invited lecture, which was presented by Beverley McKeon at the 64th Annual Meeting of the Division of Fluid Dynamics of the American Physical Society, held 20-22 November 2011 in Baltimore, MD.
} 
It is increasingly clear that turbulence is an emergent phenomenon arising from the nonlinear interaction between many different scales. As such, its analysis must be at the level of the system, such that the characteristics of the flow are considered at a global rather than local level. This makes it difficult to guarantee any global benefit of control. This may not be as difficult a problem as would be expected, since the existence of recognizable structure is suggestive that there are low-dimensional processes governing the flow. The key question is how to characterize simply these low-dimensional processes.

In recent years, dynamical systems approaches have had intriguing success in predicting the features of transition, particularly in (linearly stable) pipe flow, ${ }^{7}$ as reviewed by Eckhardt et al. ${ }^{8}$ Our understanding of transition has also benefited from a model in which small disturbances develop over infinite or finite time horizons..$^{9-11}$ In the latter picture, the system is explored by investigating the properties of the linearized Navier-Stokes system, via either its linear stability (eigenvalues) or more recently by a transient growth analysis (which investigates perturbation growth on a finite instead of infinite time horizon). These analyses are linear in nature, though often they go on to consider secondary instabilities arising from the nonlinearity.

One problem with applying perturbation analysis to turbulence is the selection of an appropriate base flow around which to linearize, since the turbulent mean velocity profile does not constitute a solution of the Navier-Stokes equations (NSE) and the transport due to turbulent fluctuations must be treated explicitly. However there have been notable efforts to extend this analysis to characterize stability and transient growth in turbulence, including the works of Butler and Farrell, ${ }^{12}$ del Álamo and Jiménez, ${ }^{13}$ Cossu, Pujals, and Depardon, ${ }^{14}$ and Hwang and Cossu, ${ }^{15}$ who used a turbulent eddy viscosity to circumvent the closure problem. The use of transient growth methodology in transitional flows is certainly well-motivated. However, despite some informative results, particularly with respect to the match between the most amplified spanwise scales (occurring for streamwise-constant disturbances) and experimental observations, ${ }^{13,15}$ the analysis of infinitesimal perturbations growing in finite time in a system where nonlinear feedback is so prevalent struck us as problematic and motivated the desire to look beyond the time-domain behaviors of unforced perturbations. Turbulent flows experience very large instantaneous deviations from any known fixed point solution and the largest growing initial condition under some time horizon will surely itself be perturbed before it reaches maturity. We suggest that things look simpler in the frequency domain.

Important steps were made in this direction by, among others, Bamieh and Dahleh ${ }^{16}$ and Jovanovic and Bamieh. ${ }^{17,18}$ There is a link, via the pseudospectrum, between the non-normality investigated in the transient growth body of work and the resolvent norm, which we will describe further in what follows. ${ }^{1,9,11}$ Those studies of the non-normality of the linearized Navier-Stokes operator, however, did not go so far as to develop a full, self-consistent approach to wall-bounded turbulence, in the sense that links to experimental observations were not made and the connection with the omitted nonlinear terms was not established.

The nonlinear triadic interaction between spatial wavenumbers in homogeneous turbulence is well-known and has been described by, among many others, Waleffe. ${ }^{19}$ There is a comparable process in frequency space, suggesting that perhaps things are not so complicated in wavenumber/frequency space. In this space, a "gain" analysis becomes natural, where wavelike motions-excited by others, via the nonlinear term-in a network of such motions, extract energy from the mean flow via a linear process and amplify disturbances through a linear resonance mechanism. The inhomogeneity in the wall-normal direction caused by the presence of the wall presents an important, but surmountable, complication of this approach in terms of the requirement on spatial collocation of interacting waves. The picture at each wavenumber/frequency combination is essentially that sketched in Figure 1, in which the lower block represents the linear dynamics of fluctuations around the turbulent mean velocity profile associated with the selective response of the resolvent discussed in Sec. II, forced by nonlinear triadic interactions from other wavenumbers and frequencies.

At the heart of the theory reported in this paper are two insights. First, that turbulence is robust. Second, that the robustness comes from a feedback loop involving the Navier-Stokes nonlinearity, which is energy-conserving, and a highly selective linear system, which leads to highly directional amplification. 


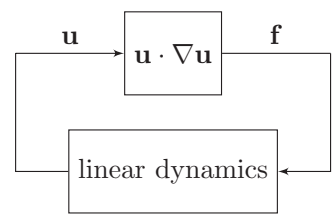

FIG. 1. A high-level description of the turbulence process. The lower block contains the linear dynamics of the fluctuations interacting with the mean velocity profile.

Robustness may be defined as the ability of a system to operate in a similar way under a wide range of conditions and uncertainties. Wall turbulence is nothing if not robust, indeed, it is the persistence of complex, but recognizable, features that characterizes these flows as turbulent. Essentially, then, in seeking to understand the processes that characterize wall turbulence, we are seeking to explain its robustness. This is not to say that "turbulence is linear," but rather that the processes that drive turbulence and select structure do appear to have a strong linear flavor (as hinted at by other studies ${ }^{20}$ ) once the nonlinear effects are confined to the determination of the mean velocity and a conservative nonlinearity.

Systems theory is rich in elegant theoretical tools to analyze and design robustness, ${ }^{21,22}$ to some large extent, that extant body of work has influenced the direction taken in this study. Inspired by these tools, we approach the turbulent flow as a system, to give us information about how the system behaves as a whole, inclusive of the nonlinearity and with a view to making direct connections to experimental and numerical observations of wall turbulence.

The full nonlinear behavior of a turbulent flow is probably too complicated to model in a simple manner, because the interconnection between scales governed by the nonlinear term would require modeling all scales at once. One approach, then, is to pretend we know nothing of the nonlinear interactions between scales and concentrate on the part we can most easily analyze, the linearized system. However, in our case, the nonlinearity is not assumed away; instead, we treat it as an unstructured forcing that has already acted to support an (assumed) turbulent mean profile of the appropriate form. The resulting decomposition of the NSE as a linear system driven by an unknown nonlinear term works because the linearized system is so selective that the exact form of the forcing is almost unimportant. We will expand on this theme throughout the paper.

A further benefit of the systems paradigm advocated here is that closed-loop control thinking and technology will carry over in the future for control designs, offering the opportunity for sophisticated closed-loop strategies (effectively based on a perfect-system model), different from the open-loop "manipulation" described here in Sec. IV. By exciting disturbances innate to the flow system, control with efficient input effort is likely achievable. The black box approach has implicitly underpinned much earlier work attempting passive and open loop control of wall turbulence, however closed loop control approaches in which the details of the model are implicit and/or imperfect have often been confounded by physical actuation and sensing requirements. Gad-el $\mathrm{Hak}^{3}$ estimates that targeting the elimination of all near-wall streaks on a typical commercial aircraft would require sensors and actuators with a frequency response of tens of kiloHertz, spaced with a density greater than 1 million per square meter. Scaled up to complete coverage of an Airbus A380, this would require an astounding $20 \times 10^{6}$ microsensors and actuators with the associated power requirements and practical control problems. To put this in perspective, the average human brain is estimated to contain (80-100) $\times$ $10^{6}$ neurons ${ }^{23}$ firing at frequencies of order $1 \mathrm{kHz}$, such that the idea of attempting real-world flow control with traditional techniques gives the concept of "fly-by-wire" an entirely new meaning!

We review here recent developments with respect to a formulation of the NSE which is amenable to systems analysis. Some new insights will be presented. The resolvent analysis developed by McKeon and Sharma, ${ }^{1}$ which will be described in detail in Sec. II below, provides, first, an exact representation of the NSE in which the output from a linear sub-system at each wavenumber/frequency combination interacts with all others to provide forcing to all linear sub-systems. The operator in each linear sub-system, called the resolvent, describes the transfer of energy into the turbulent fluctuations, along with one describing the sustenance of the mean profile that appears in all the resolvents. The inter-wavenumber energy transfer then follows from the nonlinear term as a 
consequence of gradients in energy distribution and triadic spectral interaction mechanisms. In this sense, the resolvent formulation serves as a road map for the energy transfer in wall turbulence, and captures key dynamical processes. Such information makes possible theoretical extensions to the basic framework, in order to predict the influence of prescribed external inputs and to design forcing strategies for specific, application-optimized modification of turbulent spectra. Second, the formulation admits significant mathematical simplification associated with the low-rank nature of the resolvent, which can be exploited to enable conceptually and numerically simple interrogation, replication and extension of known results in wall turbulence, ranging from the statistical to structural.

In what follows, we present an integrated picture of the power of the resolvent analysis. The exposition will be phenomenologically driven, with a view to giving an overview of the broad potential of this approach for understanding wall turbulence and ways to control it. We outline the underlying analysis and demonstrate its post- and pre-dictive power with respect to observations of real turbulence in pipe flow in Sec. III, reviewing the key results in the literature as they are introduced. Strategies for control based on the approach are outlined in Sec. IV, with a review of the first experimental demonstration of experimental manipulation of a zero pressure gradient turbulent boundary layer (ZPGTBL) using practical, wall-based, dynamic roughness actuation and use of the analysis to predict the experimental results. A brief summary and outlook concludes the paper. The material discussed here represents an integration and expansion of the work presented in assembled papers from this group, especially McKeon and Sharma, ${ }^{1}$ Jacobi and McKeon, ${ }^{24}$ and Sharma and McKeon, ${ }^{25}$ where full details of the underpinning analytical and experimental studies can be found. An abbreviated version was presented as an invited lecture during the 2011 APS-DFD meeting. ${ }^{26}$

\section{TURBULENCE AS A DIRECTIONAL AMPLIFIER}

The full analysis described in McKeon and Sharma ${ }^{1}$ was performed for pipe flow primarily because of the geometrical simplicity, the availability of high Reynolds number experimental data for comparison of statistical results ${ }^{27,28}$ and the potential for extrapolation to other canonical flows. Turbulent flow through pipes is, of course, important for the transport of fluids such as oil and natural gas, numerous natural and biomedical applications, and is also highly relevant to the study of other canonical flows. Transition to turbulence in a pipe is still not completely understood, ${ }^{29}$ but the pipe offers the analytical benefits of statistical homogeneity in the streamwise direction and a simple constraint on the azimuthal wavenumber. The coordinate system in a long, straight pipe of circular cross section for the following analysis is shown in Figure 2.

The conceptual picture underlying our analysis is that of a nonlinearity "feeding back" to excite the linear wave propagation dynamics, which in turn drive the nonlinearity (Figure 1). As such, we concentrate on the linear amplification aspect of the turbulent process and examine the corresponding linear transfer function, considering its action at each wavenumber/frequency combination (described below), which is shown to provide highly directional amplification. This picture is closely related to, but distinct from, the sector-bounding analysis of the NSE for control ${ }^{30}$ and model reduction. ${ }^{31}$

A full solution of the NSE would predict the turbulent mean velocity profile. However, a significant restriction on the scope of our modeling efforts is obtained by assuming the mean profile is known and concentrating on the action of the resolvent operators in which it appears. With

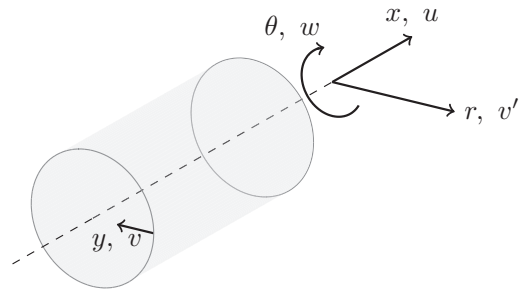

FIG. 2. A schematic of pipe geometry and nomenclature. 
the particular choice of pipe flow, experimental measurements from the Princeton Superpipe are available up to conditions representative of high Reynolds number applications ${ }^{27}$ and the use of a turbulent eddy viscosity can be avoided.

Pipe flow affords the simplification of a priori knowledge that the optimal bases for modeling the flow field in the homogeneous directions are the eigenmodes, which are the Fourier modes, such that the flow can be represented as a summation of finite-amplitude traveling wave fluctuations over a range of two-dimensional spatial wavenumbers and temporal frequencies about the turbulent mean profile, with a natural connection to the gain analysis described previously. Another obvious attraction of Fourier decomposition is the direct connection to the spectral measurements typically made in real flows. At its heart, the analysis that follows seeks to establish the optimal basis for decomposition in the inhomogeneous wall-normal direction, or the radial coherence associated with particular traveling waves. The analysis has been compared to proper orthogonal decomposition, which is a well-known technique for decomposing velocity field data to optimally capture the energy. While there is a superficial correspondence, our analysis differs in that the decomposition is predictive, since it is applied to the NSE and not pre-existing data.

\section{A. Formulation for the analysis}

Our analysis begins with the non-dimensional NSE for fully developed, incompressible pipe,

$$
\partial_{t} \tilde{\mathbf{u}}=-\nabla p-\tilde{\mathbf{u}} \cdot \nabla \tilde{\mathbf{u}}+\operatorname{Re}^{-1} \nabla^{2} \tilde{\mathbf{u}}, \quad \nabla \cdot \tilde{\mathbf{u}}=0 .
$$

We retain the boundary layer terminology by fixing $y=1-r$, and $u, v\left(=-v^{\prime}\right)$ and $w$ as corresponding to the streamwise, wall-normal and azimuthal velocities such that $\tilde{\mathbf{u}}=\left(v^{\prime}, w, u\right)$, as shown in Figure 2. The Reynolds number is defined as

$$
R e=\frac{U_{\mathrm{bulk}} D}{v}
$$

where $v$ is a constant viscosity, $D$ is the pipe diameter (equal to $2 R$ ), and the bulk, volume-averaged velocity is $U_{\text {bulk. }}$. We also introduce the Karman number,

$$
R^{+}=\frac{D u_{\tau}}{2 v}
$$

Here, $u_{\tau}=\sqrt{\tau_{w} / \rho}$ is the friction velocity, $\tau_{w}$ is the mean wall shear stress, and $\rho$ is the density.

The NSE are invariant under translation in $t$ (time), $x$ and $\theta$. In view of these symmetries, we Fourier transform in time and the two homogeneous spatial directions, introducing for convenience the wavenumber/wavespeed triplet $K=(k, n, c)$ with $k=k^{\prime} R, n=n^{\prime} R$ and wavespeed $c=\omega / k$, with $\omega=\omega^{\prime} R /\left.\mathbf{u}_{0}\right|_{y^{+}=R^{+}}$(where the prime denotes the dimensional variables and $\left.\mathbf{u}_{0}\right|_{y^{+}=R^{+}}$is the centerline velocity), and let $K \cdot \mathbf{x}=k x+n \theta-\omega t$ (notationally convenient). In addition, we specially define $K_{0}$ as $(k, n, \omega)=(0,0,0)$ and note that $\mathbf{u}_{K_{0}}\left(\mathbf{u}_{0}\right)$ is the turbulent velocity field averaged over space and time. Thus $K$ with all positive elements refers to a downstream traveling wave (helical in the cylindrical pipe geometry). As yet, only the wall-normal direction remains untransformed; the problem is to find a suitable basis for these functions of $r$. The velocity field (and forcing field) is expressed as a sum of harmonic, radially varying traveling waves,

$$
\mathbf{u}(r, x, \theta, t)=\sum_{n} \int_{-\infty}^{\infty} \int_{-\infty}^{\infty} \mathbf{u}_{K}(r) e^{i K \cdot \mathbf{x}} \mathrm{d} k \mathrm{~d} \omega .
$$

We define $\mathbf{u}$ and $\mathbf{f}$ as

$$
\begin{gathered}
\mathbf{u}=\tilde{\mathbf{u}}-\mathbf{u}_{0}, \\
\mathbf{f}=-\mathbf{u} \cdot \nabla \mathbf{u},
\end{gathered}
$$

with $\mathbf{u}_{K}(r)$ and $\mathbf{f}_{K}(r)$ the (radially varying) Fourier coefficients thereof. 
The NSE assuming fully developed flow (i.e., real elements of $K$ ) can then be written in a divergence-free basis as

$$
\begin{aligned}
-i \omega \mathbf{u}_{K} & =\mathcal{L}_{K} \mathbf{u}_{K}+\mathbf{f}_{K}, \quad(k, n, \omega) \neq(0,0,0), \\
0 & =\mathbf{f}_{0}-\mathbf{u}_{0} \cdot \nabla \mathbf{u}_{0}+R e^{-1} \nabla^{2} \mathbf{u}_{0} .
\end{aligned}
$$

Note that in this formulation, the linearized Navier-Stokes operator, $\mathcal{L}_{K}$, naturally involves the turbulent mean; yet we have performed no (linearized) stability analysis around a fixed point. Thus the $\mathbf{u}_{K} \mathrm{~s}$ are exactly the Fourier coefficients of the turbulent fluctuations relative to the mean profile at all $y$. Previous work concerning analysis of $\mathcal{L}_{K}$ and globally optimal perturbations has typically employed a turbulent viscosity to account for the turbulent transport that would be excluded by a linearization procedure. In this analysis, the treatment of $\mathbf{f}$ explicitly accounts for this phenomenon, so the resolvent contains only the turbulent mean velocity profile itself.

A trivial manipulation of Eq. (5) gives the response of the flow at a particular wavenumber combination subjected to harmonic forcing $\mathbf{f}_{K}$ arising from the interaction between other wavenumbers,

$$
\mathbf{u}_{K}=\mathcal{H}_{K} \mathbf{f}_{K}=\left(-i \omega I-\mathcal{L}_{K}\right)^{-1} \mathbf{f}_{K},
$$

where $\mathcal{H}_{K}$ is known in the literature as the resolvent. ${ }^{32}$ Using the form for $\mathcal{L}_{K}$ given in Meseguer and Trefethen, ${ }^{33}$ we can write

$$
\mathcal{H}_{K}=\left[\begin{array}{ccc}
i\left(k \mathbf{u}_{0}-\omega\right)-R e^{-1} D & -2 i n r^{-2} R e^{-1} & 0 \\
2 i n r^{-2} R e^{-1} & i\left(k \mathbf{u}_{0}-\omega\right)-R e^{-1} D & 0 \\
-\partial_{r} \mathbf{u}_{0} & 0 & i\left(k \mathbf{u}_{0}-\omega\right)-R e^{-1}\left(D+r^{-2}\right)
\end{array}\right]^{-1}
$$

with $D=\partial_{r}^{2}+r^{-1} \partial_{r}-r^{-2}\left(n^{2}+1\right)-k^{2}$, and the states being the radial, azimuthal, and axial velocities expressed in a divergence-free basis. Thus Eqs. (5) and (6) can be visualized as the block diagram in Figure 3, forming a complete representation of the NSE in this basis. The resolvents, $\mathcal{H}_{K}$, can be derived from the NSE and are interconnected through the nonlinear terms. Note that (as stated in the caption) while an input-output relationship can be written for all $K, \mathbf{u}_{K} \neq 0$ for a finite range of $K$ in real flows.

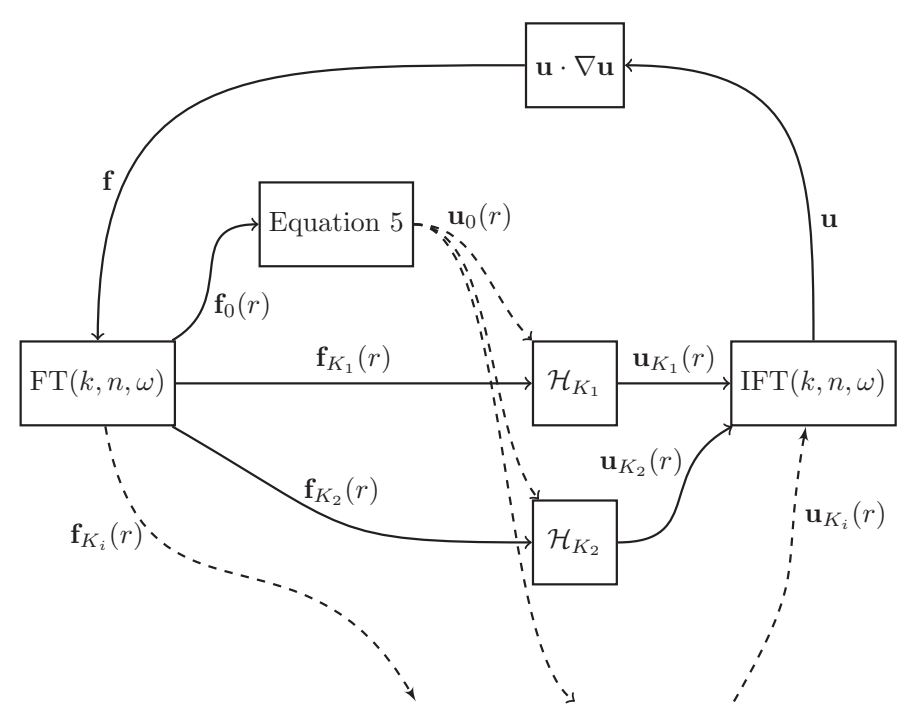

FIG. 3. A block diagram showing the network of resolvents, $\mathcal{H}_{K}$, acting on radially varying traveling waves of different wavenumber and frequency. An input-output relationship can be written for all $K$, but $\mathbf{u}_{K} \neq 0$ for a finite range of $K$ in real flows. FT and IFT denote the Fourier transform and inverse Fourier transform, respectively. $u_{0}$ and $f_{0}$ describe the equation for the turbulent mean profile. 
Although the resolvents are linear, the nonlinear forcing internal to the system is explicitly retained so this is not a linearized analysis. Additional effects of the nonlinearity are bound up in the use of the turbulent mean velocity profile in the resolvent. Thus the analysis is fundamentally different from linear stability analysis: it concerns the response of a forced system (in reality lightly forced because the leading singular values in general give rise to large amplification), where the forcing is required to sustain a response in a linearly stable system. Thus, concepts relevant to the study of disturbances in inviscid, linearized laminar flow can be extended to the turbulent case, with the understanding that in the latter case the waves are lightly damped and would asymptotically decay in the absence of forcing $\mathbf{f}$.

Figure 3 describes an interconnected series of linear sub-systems with resolvents of known form. Thus the formulation of Eqs. (5) and (6) lends itself naturally to separate analysis of the individual sub-systems associated with each $K$, under the constraint that the correct amplitudes for each $\mathbf{u}_{K}$ are required to sustain the (assumed) mean velocity profile in the fully connected system. Without satisfying this constraint associated with the excitation of all $\mathbf{u}_{K}$ through the nonlinear term, progress can still be made in terms of determining the radial distributions of velocity for each $K$, since the linearity of the system allows for simple amplitude scaling of the response mode shapes. In effect, then, we can consider unstructured (unit) forcing of each sub-system, in knowledge that the output response modes can be linearly superposed to return to a representation of the full velocity field subject to the constraint that the response mode amplitudes must ultimately satisfy Figure 3 . This is another significant advantage of our approach: the ability to dissect the flow into manageable "lumps" (linear sub-systems) that can be analyzed in isolation and then simply reassembled. Further, this formulation proves amenable to approximation of the resolvent at each $K$ that leads to a significant reduction in complexity using standard linear systems techniques.

\section{B. Optimal low-rank approximation of the resolvent for inhomogeneous coordinates}

The symmetries associated with the pipe geometry imply that the resolvent is normal under the integrals over the wall-parallel coordinates and time (see Sharma and Mckeon ${ }^{25}$ for further discussion on this point). In these homogeneous directions, then, the forcing and response modes are equal, orthogonal and are the Fourier modes, which then represent the appropriate choice for basis functions. However, this is not true in the wall-normal direction: the presence of the wall leads to the loss of symmetry with the implication that the forcing and response modes are no longer equal and the fluctuations may now gain energy from the interaction with the mean flow. The potential for momentum production due to this interaction is quantified by this loss of orthogonality. Since the resolvent is not normal with respect to the integral in this direction, the Fourier basis is no longer the optimal choice under the energy norm. We seek a basis that is optimal with respect to the magnitude of response to forcing in order to continue our gain-based analysis. To find this basis, we use the singular value decomposition (SVD). For the reader less familiar with the SVD, we give a brief example of its underlying action and interpretation in the next two paragraphs which the more accustomed reader may safely skip.

The SVD is a well-known matrix decomposition that splits any matrix $\mathbf{M}$ into the product $\mathbf{M}=\mathbf{A S B} *$ where $\mathbf{A}$ is a unitary matrix $\left(\mathbf{A A}^{*}=\mathbf{A} * \mathbf{A}=\mathbf{I}\right), \mathbf{S}$ is a diagonal real matrix, and $\mathbf{B}$ is the conjugate transpose of another unitary matrix, all of appropriate dimension, as in the example of Figure 4. The diagonal elements of $\mathbf{S}, \sigma_{i}$, are ordered such that $\sigma_{i} \geq \sigma_{i+1}$ and are called the singular values. When applied to statistical data, the SVD is commonly known as principal component analysis, Karhunen-Loéve decomposition, or proper orthogonal decomposition (POD). Comparison across application domains is not very meaningful however, since the object of optimization is quite different.

The singular values contained in the diagonal matrix $\mathbf{S}$ quantify the underlying dimensionality of the mapping being decomposed. Where $\sigma_{2}$ is small compared to $\sigma_{1}$, as in the simple example in Figure 4, the full mapping of a unit circle at input to an ellipse at output is well approximated by the lower-dimensional mapping to a line. This example illustrates the use of the SVD to find lowerrank approximations to linear mappings between higher-dimensional spaces. The SVD generalizes to apply to linear operators on Hilbert spaces ${ }^{34}$ where it becomes known as the Schmidt decomposition. 

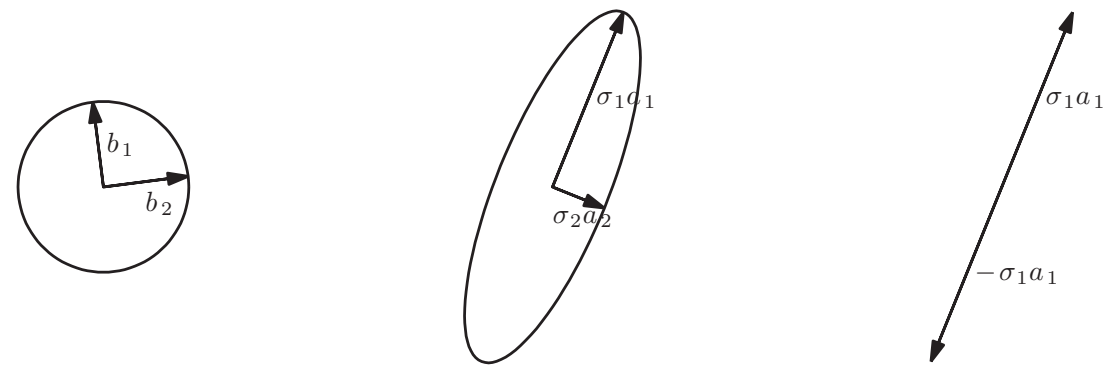

FIG. 4. An illustration of the effect of matrix $\mathbf{M}$ on the unit circle (left plot mapped to centre plot). The singular values are the radii of the resulting ellipse and the singular vectors give the rotation of the ellipse. $\mathbf{a}_{i}$ are the columns of $\mathbf{A}$ and $\mathbf{b}_{i}$ are the columns of $\mathbf{B}$. In this illustration, the matrix $\mathbf{M}=\left[\begin{array}{cc}0.5 & 0.9 \\ -0.5 & 2\end{array}\right]$ has the singular value decomposition $\mathbf{M}=\mathbf{A S B}^{*}$ with $\mathbf{A}=\left[\begin{array}{cc}0.3761 & 0.9266 \\ 0.9266 & -0.3761\end{array}\right], \quad \mathbf{S}=\left[\begin{array}{cc}2.2089 & 0 \\ 0 & 0.6564\end{array}\right], \quad \mathbf{B}=\left[\begin{array}{cc}-0.1246 & 0.9922 \\ 0.9922 & 0.1246\end{array}\right]$. The rightmost plot shows the effect of the optimal rank-1 approximation $\tilde{\mathbf{M}}$ to the mapping $\mathbf{M}$ on the unit circle, where $\tilde{\mathbf{M}}=\mathbf{A}\left[\begin{array}{cc}2.2089 & 0 \\ 0 & 0\end{array}\right] \mathbf{B}^{*}$.

Hopefully by now the application of the SVD in our context becomes obvious: since we hold that turbulence can be captured by a low-order amplifying process, we must find the "best" low-rank approximation to the resolvent, where what is meant by "best" is quantifiable in a defined sense. The result is simply a truncation of the Schmidt decomposition of the resolvent, or the identification of the right singular vectors (inputs) that give rise to the most amplified left singular vectors (outputs). The relative amplification factor for unit input amplitude is given by the associated singular value. We term the left and right singular vectors the velocity response and forcing modes, respectively; the first singular forcing modes are the most significant in that they can be expected to dominate the full observed response at a given $K$ if the resolvent is truly low rank. We confirm this assumption in the results shown below, noting that the true output from each resolvent will be the product of forcing amplitude and singular value.

The SVD of the resolvent before Fourier decomposition is imposed would itself naturally result in a decomposition into the (optimal) Fourier basis in the symmetric directions for the reasons discussed earlier. For simplicity, we take that stage as given and consider the optimal low rank approximation only in the inhomogeneous, wall-normal direction.

The SVD of the resolvent for a particular $K$ can be written in terms of the forcing $(\phi)$ and response $(\psi)$ modes associated with the response of the flow as follows:

$$
\left(-i \omega I-\mathcal{L}_{K}\right)^{-1}=\sum_{l=1}^{\infty} \psi_{l K}(r) \sigma_{l K} \phi_{l K}^{*}(r)
$$

with the orthogonality condition

$$
\left(\phi_{l K}(r), \phi_{m K}(r)\right)_{r}=\delta_{l m}, \quad\left(\psi_{l K}(r), \psi_{m K}(r)\right)_{r}=\delta_{l m},
$$

where $(\cdot, \cdot)_{r}$ indicates the inner product over $r$, such that the sets of both forcing and response modes are normalized with respect to the energy integrated over the radius of the pipe and are orthogonal. The singular values are sorted, with $\sigma_{l, K} \geq \sigma_{l+1, K} \geq 0$. For stable $\mathcal{L}_{K}$, this decomposition exists for real $\omega$. It is also unique up to a pre-multiplying unitary complex factor on both bases corresponding to a phase shift and up to the ordering in $l$ of $\sigma_{l K}$ s, hence we fix the relative phases with respect to the first coefficient. 


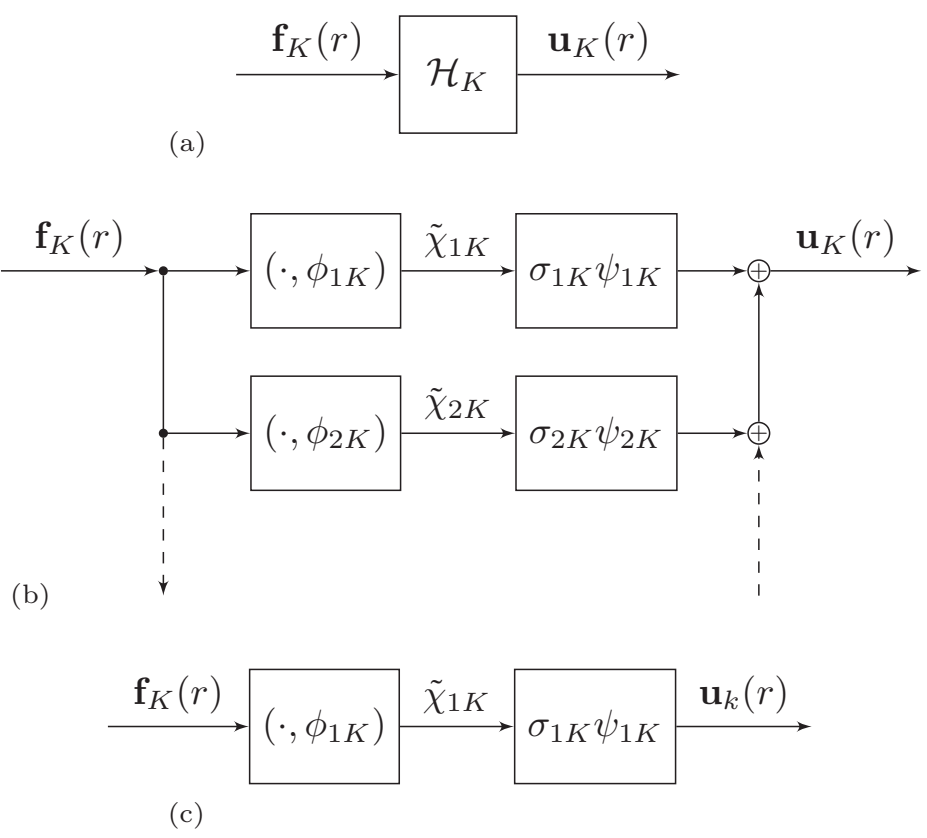

FIG. 5. Block diagram for Eqs. (11) and (12) referring to the linear equations projected onto the forcing and response modes. Diagram (a) depicts the "true" resolvent and (b) depicts the Schmidt decomposition of the resolvent. The rank-1 approximation is depicted in (c). This approximation can be performed for each lower sub-system in Figure 3.

The basis pair defined by $\phi_{I K}$ and $\psi_{I K}$ can be used to decompose arbitrary forcing and the resulting velocity at any particular frequency/wavenumber component $K$, such that

$$
\begin{gathered}
\mathbf{f}_{K}(r)=\sum_{l=1}^{\infty} \phi_{l K}(r) \tilde{\chi}_{l K}, \\
\mathbf{u}_{K}(r)=\sum_{l=1}^{\infty} \sigma_{l K} \psi_{l K}(r) \tilde{\chi}_{l K},
\end{gathered}
$$

where the $\tilde{\chi}$ 's correspond to the coefficients of projection of the forcing onto the forcing modes.

The radial shape of harmonic forcing that gives the largest disturbance energy (given unit forcing amplitude) at a particular $K$ is $\mathbf{f}_{K}=\phi_{1 K}$, with a gain of $\sigma_{1 K}$. The next largest arises from $\mathbf{f}_{K}=\phi_{2 K}$ and so on, at a particular wavenumber pair and frequency. The corresponding flow response modes are given by the related $\mathbf{u}_{K}=\psi_{1 K}, \psi_{2 K}$, etc. This decomposition of general input forcing is illustrated in Figures 5(a) and 5(b).

At this stage, the analysis is complete in the divergence-free basis, in the sense that no modeling assumptions have been made, other than that the velocity field is statistically homogeneous in $x, \theta$, and $t$. Linearity of the system implies that the resolvent can be analyzed under unstructured forcing, i.e., isolating individual resolvents as shown in Figure 5(a), but the appropriate amplitudes of the velocity response modes must be determined before the connected, self-sustaining representation of Figure 3 can be reassembled (the topic of ongoing work). The optimal low-rank approximation to the resolvent is defined to arbitrary accuracy related to the number of singular modes included. There is a further technical assumption that the resolvent is compact. The simplest approximation is rank-1, in which the resolvent is modeled using only the first singular modes (Figure 5(c)); this approximation is likely to be good if $\sigma_{1 K} \gg \sigma_{I K}$ for all $l>1$. While the assumption that a rank-1 approximation is sufficient to model wall turbulence is quite strong to begin with, it is one of the key outcomes of this formulation that it yields surprisingly good agreement with observations from real flows for the vast majority of observed $K$ combinations. Of course, a higher-rank approximation may simply be made by the same process, should higher fidelity be required. 


\section{Response of radially varying traveling waves and the connection with critical layer theory}

The singular response modes were computed using a MATLAB implementation of the decomposition described in equations in Sec. II B and the numerical scheme and divergence-free basis for pipe flow proposed by Meseguer and Trefethen, ${ }^{33}$ modified for a turbulent mean flow as in McKeon and Sharma. ${ }^{1}$ Full details of the computational approach can be found in these two papers and, for brevity, are not repeated here. The computational expense is exceedingly low, even for analysis of Reynolds number up to $R^{+}=1.97 \times 10^{4}$, the highest $R e$ study we have performed to date.

McKeon and Sharma ${ }^{1}$ investigated the variation of the characteristics of the radially varying traveling waves over a range of $K$ and made some observations with respect to variation with singular value. Recall that the $\sigma_{l}$ gives the amplification, and complex functions $\phi_{l}$ and $\psi_{l}$ give the wall-normal variation of the amplitude and phase of the forcing and response mode, respectively (where the phase of the response mode is relative to the forcing). In particular, the orthogonality requirements given in Eq. (10) require an increase in the number of maxima in the radial amplitude function $\mathbf{u}_{K}(\mathrm{r})$ with increasing $l$ (with analogy to the orthogonality of Fourier modes), as shown in the comparison of velocity response modes with increasing $l$ in Figure 2 of McKeon and Sharma. ${ }^{1}$ (An example of the variation of singular values with $l$ is shown in Figure 3 of that paper.) The combination of the increasing complexity of such structures and the rapid drop-off in singular value observed with increasing $l$ support the rank-1 hypothesis for a wide range of $K$; in the rest of this paper, we consider only the output of the rank-1 model of the NSE at each $K$ value (Figure 5(c)), which we call the first velocity response modes.

We summarize the general features of the first velocity response modes in Figures 6 and 7 , for a particular $(k, n)=(1,10)$ at $R^{+}=1800$. Figure 6(a) shows the wall-normal variation of the location of the peak magnitude of the streamwise velocity perturbation with increasing wavespeed $c$, compared with the local turbulent mean velocity profile $\mathbf{u}_{0}$. The variation with $c$ of the associated first singular value, $\sigma_{1}$ is shown in Figure 6(b); note that for this $K$ (and a wide range of $K$ in general), the magnitude of the first singular value is extremely large, meaning that only very small forcing amplitude will be required to observe a strong velocity response.

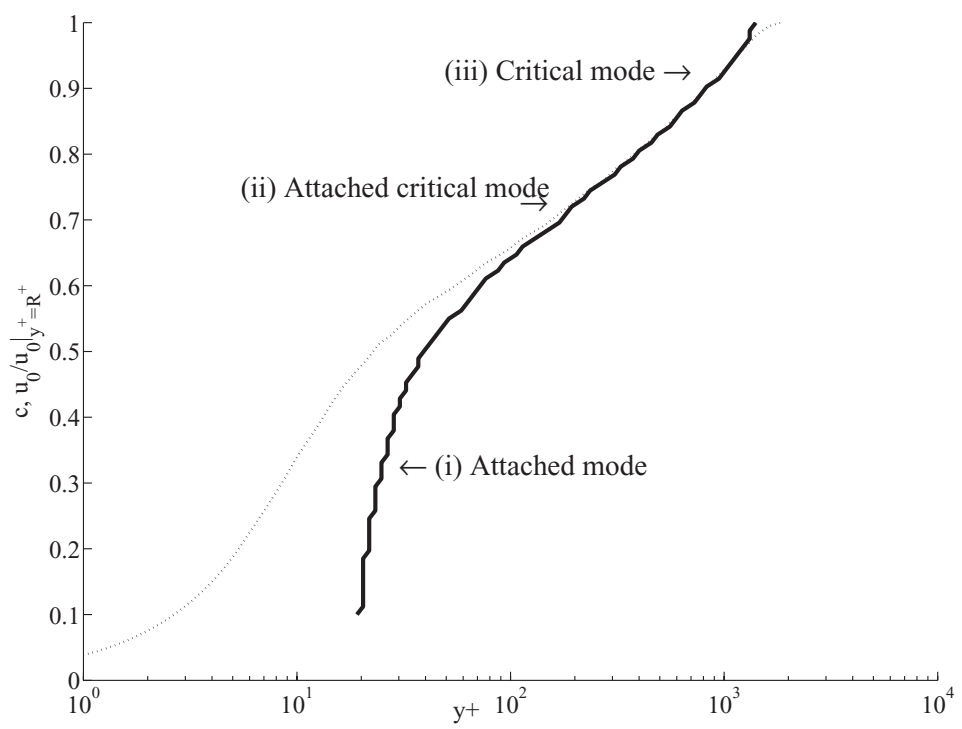

(a)

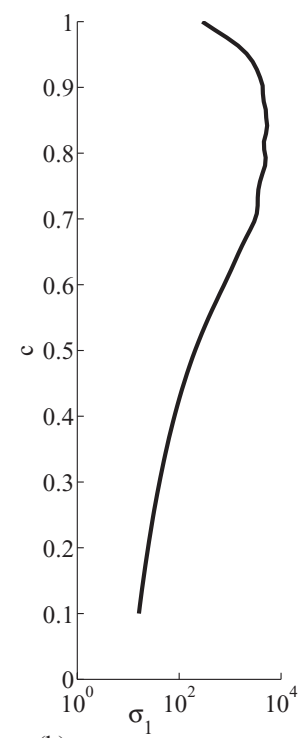

(b)

FIG. 6. (a) Variation of the location of the peak streamwise velocity with increasing wavespeed, $c$, for the first velocity response mode with $(k, n)=(1,10)$ at $R^{+}=1800$ (solid line). The turbulent mean profile normalized by the centerline velocity, $\mathbf{u}_{0} /\left.\mathbf{u}_{0}\right|_{y^{+}=R^{+}}$, at this Reynolds number is also shown in dotted gray for reference. (b) The corresponding first singular values. 

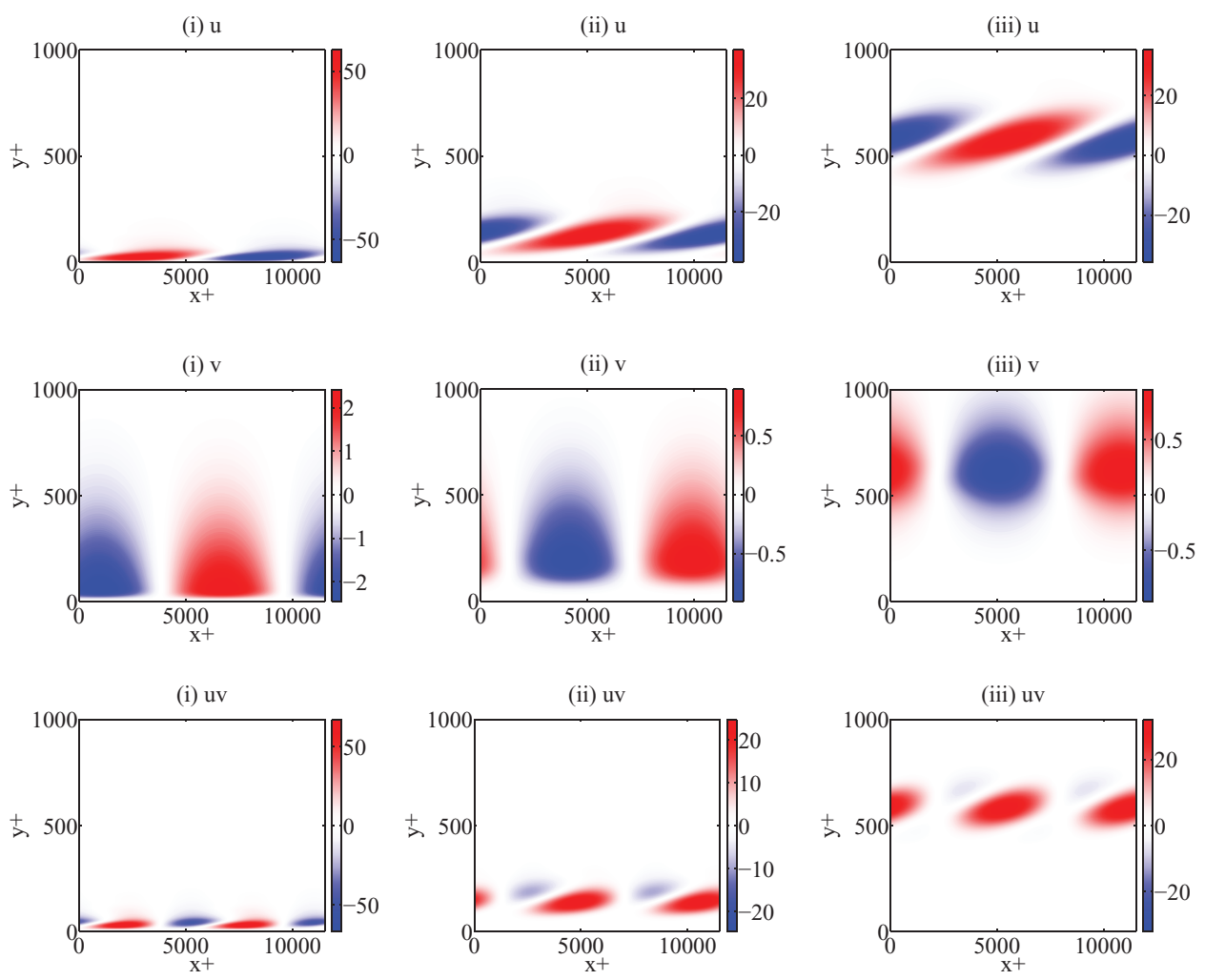

FIG. 7. Representative velocity response mode shapes for the "attached," "attached and critical," and "critical modes," shown for conditions corresponding to points (i)-(iii) in Figure 6, i.e., $(k, n)=(1,10), c=0.25,0.67$ and 0.85 , and $R^{+}=1800$. (Top) $u\left(y^{+}\right)$; (middle) $v\left(y^{+}\right)$; (bottom) $u v\left(y^{+}\right)$.

Three different types of modes can be identified in Figure 6(a). The velocity response mode shapes $\mathbf{u}_{K}$ corresponding to these three classes of velocity response modes, identified here by (i)-(iii), are shown in Figure 7. The variation of the azimuthal component is not shown since it is simply determined from the summation of left- and right-going propagating waves described earlier and the continuity equation, and is out of phase with $\mathrm{u}$ in both streamwise and azimuthal directions. When $c$ is small in region (i), the peak magnitude in all components stays at an approximately constant wall-normal distance, $y^{+}=y u_{\tau} / \nu$. We term these "attached modes," because the footprints of the modes reach down to the wall. For large $c$ in region (iii), the location of the peak amplitude tracks the local mean velocity, i.e., $c=\mathbf{u}_{0}(y)$ and there exists a critical layer. The mode is localized around the critical layer, such that these "critical modes" rapidly become detached from the wall with increasing $c$. Some special significance will be given to the class of attached, critical modes (ii), i.e., the slowest convecting modes that achieve critical status.

Note also that the Reynolds stress associated with the velocity response modes exhibits slightly different behavior, lifting from the wall to provide a radially localized distribution with contributions at $K_{0}$ and $2 K$.

We will not comment here on the first singular forcing mode shapes corresponding to the velocity response modes shown in Figure 7, beyond saying that the forcing occurs locally to the velocity response in a radial sense, and that understanding which pairs of other $K$ s supply a component of forcing in these directions is equivalent to understanding how to close the block diagram of Figure 3.

The physics underlying these distinct classes of response modes can be identified by returning to the full rank resolvent of Eqs. (7) and (8) (and noting that the scaling phenomena observed are reflected in the rank-1 approximation). There are two distinct mechanisms by which the resolvent can lead to large amplification: ${ }^{1}$ the non-normality associated with the $v \partial_{r} \mathbf{u}_{0}$ term coupling the 
radial perturbation to the mean shear (requiring a mode covering a wider range in the wall-normal direction, since it is powered by the differential in velocity over $r$ ), and a critical-layer type response where locally $c \simeq \mathbf{u}_{0}\left(y_{c}\right)$ giving high gain. The former effect is well understood to lead to energy extraction from the mean flow under other formulations. In the latter case, the diagonal terms in the resolvent become extremely large at the location where $c=\mathbf{u}_{0}(y)$ with large Reynolds number. The concept of a critical layer in wall turbulence has received much less attention, with the notable exception of the analysis by Sreenivasan. ${ }^{35}$ This phenomenon occurs when the resolvent at this location becomes large because, at high enough Reynolds number, the response at the critical layer to forcing is almost singular; the relevant eigenmodes approach neutral stability in the inviscid limit.

A review of the body of work on critical layers in laminar flow is given by Maslowe $\mathrm{e}^{36}$ and Schmid and Henningson. ${ }^{11}$ There are some essential differences to the current development: we address the turbulent case, we maintain the presence of the nonlinear forcing term and (specific to pipe flow) the wall-normal velocity and vorticity in the Orr-Sommerfeld formulation of the problem do not decouple in cylindrical coordinates. ${ }^{11}$ However critical layer concepts are useful, and qualitative connections are made between the theory and turbulent flow in Cartesian geometries in McKeon and Sharma. ${ }^{1}$

\section{Closing the loop: An explicit treatment of the nonlinearity}

The linear selection mechanism explains much of the form of the structure of real flow; however the nonlinearity is also important. Though it is not the focus of this work, we will briefly discuss it here for the case of a discretised field, i.e., discrete $K$. Using the resolvent decomposition of Eqs. (7), (9), (11), and (12), expressing the fields in terms of the coefficients with $j, a, b$ indexing over all $l K$, we get

$$
\mathbf{u}=\sum_{j} \chi_{j} \psi_{j}=\sum_{j} \psi_{j} \sigma_{j} \phi_{j}^{*} f_{j} \phi_{j}
$$

where

$$
f_{j}=\sum_{a, b}\left(-\psi_{a} \cdot \nabla \psi_{b}, \phi_{j}\right)_{r} \chi_{a} \chi_{b}=\sum_{a, b} N_{j a b} \chi_{a} \chi_{b}
$$

Here, $\chi_{j}$ are the coefficients describing the projection of the velocity field onto the velocity response modes, $\chi_{j}=\sigma_{j} \tilde{\chi}_{j}$, and $N_{j a b}$ is the coupling between any three traveling wave singular modes. Note that $N_{j a b}=0$ where Fourier modes are triadically incompatible. The full nonlinear problem can then be reduced to the solution of

$$
\chi_{j}=\sum_{a, b} \sigma_{j} N_{j a b} \chi_{a} \chi_{b} .
$$

The modeling question is the magnitude and sparseness of both $N_{j a b}$ and $\sigma_{j}$. Notice that high gain $\sigma_{j}$ can compensate for low (but non-zero) $N_{j a b}$ and the quadratic property of $\mathbf{f}$ can determine the amplitudes. Since $\sigma_{j}$ and $N_{a j b}$ are calculated from the NSE, this system of equations together with the assumed mean velocity profile constitutes a complete description of turbulence with transient behavior removed. Sparseness makes this representation very efficient.

We see that self-sustaining mode combinations are possible if the nonlinear forcing term resulting from the interaction of component velocity modes is not orthogonal to the forcing modes required to sustain it. We could reasonably look for solutions to (15) to find self-sustaining mode combinations. Such a truncation would give a low-order, discrete representation of (15). This is the subject of ongoing work.

This view also gives an interesting insight into the equivalent of the cascade in the inhomogeneous spatial direction. In this direction, because of the distribution across $r$ of the modes, there is a non-local (distributed) interaction with the mean shear. This possibility of extracting energy from the velocity profile is mathematically captured by the non-normality. The critical layer response, by contrast, is localized in $r$. 
The triadic interaction in homogeneous isotropic turbulence (or the $t, x$, and $\theta$ directions) is well understood; the mechanisms for energy transfer have now been effectively extended to the inhomogeneous directions by the analysis. The nonlinearity, in addition to transferring energy across $\omega, k$, and $n$, subject to the rules of triadic interaction, may also transfer energy across response modes in $y$, subject to the three-way interaction $N_{j a b}$ and the singular values $\sigma_{j}$. The ability of a mode to drive others in the wall normal direction, essentially the wall-normal cascade, is determined by the localization of the mode in that direction, which in turn determines $N_{j a b}$.

\section{E. Where to look in 3D spectral space?}

In the absence of a solution to Eq. (15) that identifies the modes required to sustain the turbulence with $N_{j a b} \neq 0$, our analysis at this stage is guided by the range of $K$ observed in real flows (although note that ongoing work suggests that this region of $K$ space corresponds well to the range where the first singular values have large magnitude, e.g., Ref. 37). This localization and high response at the critical layer dictates that the turbulent flow's energy is concentrated around a thin "spine" in $(k, n, \omega$, $l$ ) space, where $l$ is the singular value index, which essentially describes a low-dimensional attractor in this space. This will also be discussed in forthcoming work. As such, we briefly review work that has characterized the scales and wall-normal variation of the three-dimensional, spatio-temporal $K$ spectrum (which in its entirety is still a relatively uncommon result) and has thus informed our work.

The statistics and spectra of the turbulent fluctuations have been historically well studied, such that results including the Reynolds-number independence of the near-wall cycle and the energetic importance of the very large scales emerging with increasing Reynolds number are now well established. However, the classification of coherent structures and their connection to velocity statistics can be said to be a more recent focus, significantly enabled by advances in computational and experimental diagnostic technology. The essential structural features of wall turbulence have been listed recently by Smits, McKeon, and Marusic. ${ }^{38}$ In order of increasing streamwise scale, this list consists of near-wall streaks of streamwise velocity, hairpin vortices, large scale motions (LSM) believed to correspond to organization of hairpin vortices into packet structures, and very large scale motions (VLSM) with streamwise extent on the order of ten times the outer lengthscale. The temporal frequency is most simply addressed through the range of convection velocities observed in the flow. ${ }^{39-42}$

Assuming the approximate universality of spectra between canonical flows documented by Monty et al. ${ }^{43}$ and the range of scales described in Smits, McKeon, and Marusic ${ }^{38}$ and references therein, conservative estimates of the energetically active $K=(k, n, c)$ ranges are then $0.1<k$ $<2 \pi R^{+} / 100,2 \pi / 0.1<n<2 \pi R^{+} / 10$, and $10 /\left.\mathbf{u}_{0}^{+}\right|_{y^{+}=R^{+}}<c<1$. Practically, the sum of $K$ and its complex conjugate, and both left- and right-going waves $( \pm n)$ must be considered in order to obtain real-valued amplitudes and avoid a non-zero mean spanwise velocity, respectively. In what follows, we constrain our interrogation of the resolvent analysis to these values and document well-known features of wall turbulence that are captured by the rank-1 approximation to the resolvent.

\section{REPRODUCTION AND EXTENSION OF STATISTICAL AND STRUCTURAL RESULTS IN UNPERTURBED WALL TURBULENCE}

An array of quantifiable features of wall turbulence have been well described in the extensive wall turbulence literature, including velocity statistics, spectral information, and organizations of coherent structure, even if their Reynolds number scaling and dynamical importance still remain sufficiently elusive to preclude effective low order modeling. Of course, even observing these features at high Reynolds number presents severe experimental and numerical challenges, but assimilation of the characteristics of all such recognizable results is required for a full understanding of wall turbulence.

Our analysis naturally leads to information related to velocity spectra and distribution of statistics in the wall-normal direction, and we will present selected results in this section. We also target the different classes of structures listed in Sec. II E above, demonstrating that the analysis has the 
potential to unify the statistical and structural observations of wall turbulence and lead to inferences about the key underlying physics.

We note that experimental studies typically interrogate data based on averaging conditional on some characteristic typical to the structure to be examined. For instance, Dennis and Nickels ${ }^{44}$ average conditional on a swirl criterion to identify hairpin vortices, yielding the scale distribution in $K$ and $y$ associated with the structure. In contrast, we work in the opposite direction: specifying $K$ and yielding the wall-normal distribution of the velocity of the response modes, which we associate with coherent structure.

\section{A. Near-wall cycle}

The structure and dominant dimensions associated with the autonomous, near-wall cycle of turbulence have been known since the seminal experiments of Kline et al. ${ }^{45}$ Significant study of the dynamics of the flow in this region, performed by, e.g., Waleffe, ${ }^{46}$ Jiménez and Pinelli, ${ }^{47}$ and Schoppa and Hussain ${ }^{48}$ using low Reynolds number data, revealed the well-known quasi-streamwise vortex and streamwise velocity streak structure, while experiments in the atmospheric surface layer ${ }^{49}$ have demonstrated the Reynolds number independence of the streak spacing in viscous units at the near-wall scales identified above, namely, $\lambda_{x}^{+} \approx 1000$ and $\lambda_{z}^{+} \approx 100$. Comparison with the structure arising from our analysis requires the specification of an additional parameter, the phase velocity, $c$. For the dominant wavelengths associated with the near-wall cycle, the first singular mode is critical and attached to the wall, as identified in Figure 7, when $c^{+}=10-15$, the minimum convection velocity associated with energetic disturbances.

The wall-normal distribution of the streamwise component of the first singular mode with $K=\left(2 \pi R^{+} / 1000,2 \pi R^{+} / 100,10 /\left.\mathbf{u}_{0}^{+}\right|_{y^{+}=R^{+}}\right)$is essentially Reynolds number-independent, as shown by the distributions of the amplitude over an order of magnitude in Reynolds number in Figure 8(a). In fact, this independence emerges directly from the Cartesian coordinate version of critical layer scaling ${ }^{1}$ for modes with $K$ constant in inner units in a region where the mean velocity profile is self-similar in inner units, i.e., for sufficiently high Reynolds numbers. The first singular velocity response mode captures this scaling and thus postdicts from a low rank approximation to the NSE that the location of the near-wall cycle activity should be Reynolds number independent. Similar results can be obtained for outer scaling modes. ${ }^{1}$

The velocity components are distributed in such a way as to create a periodic array of alternating streamwise velocity streaks accompanied by streamwise-aligned vortices in the cross-stream plane, which are elevated slightly from the wall with increasing downstream distance. See Figure 8(b) and Figure 1 from McKeon, Sharma, and Jacobi, ${ }^{50}$ for a cartoon of the structure associated with this near-wall mode. There is a strong resemblance to a system of quasi-streamwise vortices and streaks of streamwise velocity, with the simplicity of this representation made possible because of

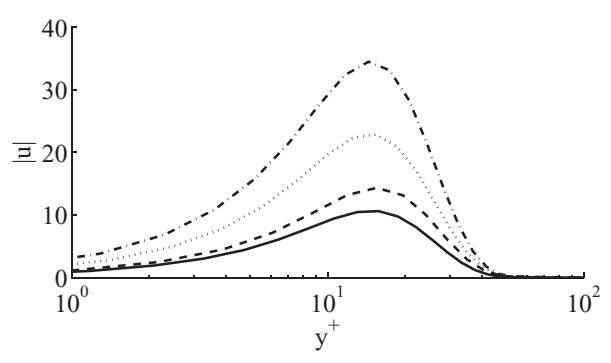

(a)

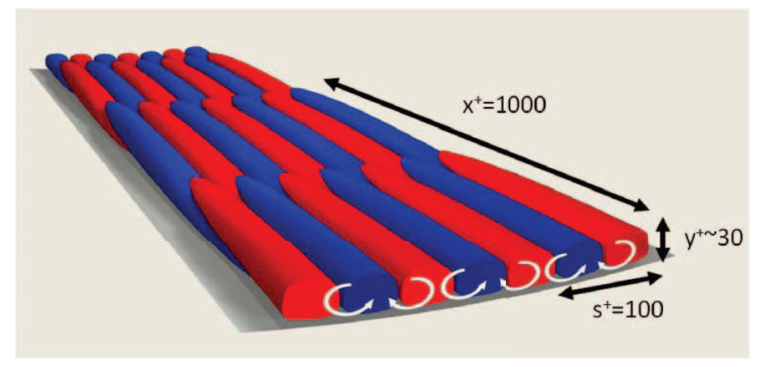

(b)

FIG. 8. (a) Distribution of streamwise energy over the pipe radius for the near wall mode with $K=\left(2 \pi R^{+} / 1000,2 \pi R^{+} / 100,10 /\left.\mathbf{u}_{0}^{+}\right|_{y^{+}=R^{+}}\right)$. Reynolds numbers: $-75 \times 10^{3},--150 \times 10^{3}, \cdots 410 \times 10^{3}, \cdot-\cdot 1 \times$ $10^{6}$. (b) Shape of the first singular mode representative of the dominant near wall motions. Color denotes isosurfaces of streamwise velocity (streaks), where red and blue correspond to high and low velocity, respectively, relative to the mean flow (heading into the page), and the white arrows show the sense of the in-plane velocity field. 


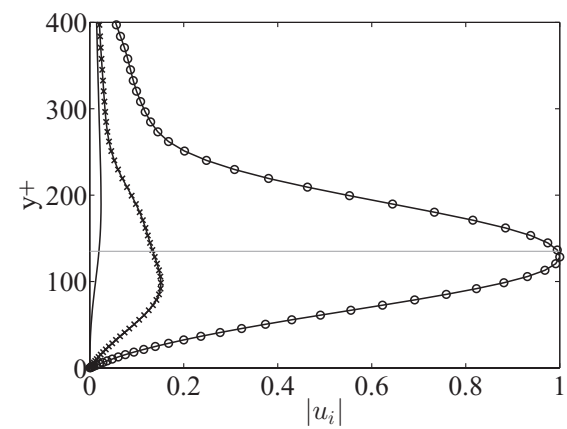

(a)

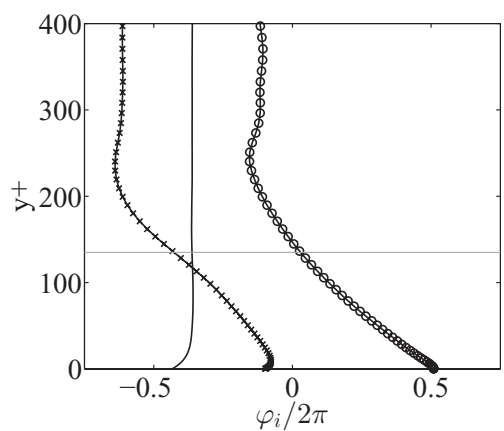

(b)

FIG. 9. Wall-normal variation of (a) amplitudes of the three velocity components and (b) corresponding phases in multiples of $2 \pi$ for the VLSM response mode with $K=(1,10,2 / 3)$ at $R^{+}=1800$. The lines denote $-o-: u,-: v,-\times-: w$. Solid/dashed horizontal line shows the location of the critical layer.

the absence of decorrelation associated with the many other (less-energetic) $K$ modes that are active in this region of the flow.

Thus we see that, first, the rank-1 approximation to the resolvent generates velocity response modes that display the scaling, distribution, and structure consistent with the state of understanding concerning the near-wall cycle. Second, a modified critical layer analysis provides scaling consistent with observations of wall turbulence.

\section{B. Characteristics of the very large scale motions}

Similar success can be obtained by studying the first singular velocity response mode at the $K$ combination believed to correspond to the VLSMs. Figure 9 shows the response mode amplitude and phase associated with $K=(1,10,2 / 3)$, selected by McKeon and Sharma ${ }^{1}$ to best match a series of observations of VLSMs in the literature that are incomplete in terms of the three parameters required for input to our analysis. Irrespective of the exact values selected, the general form of this mode is similar to the attached critical mode shown in Figure 6, namely, streaks of streamwise velocity with a small inclination from the wall and associated cross-stream vortices, in agreement with the observations of Hutchins and Marusic ${ }^{51}$ and Chung and McKeon. ${ }^{52}$ The strength of each of these features, common to attached critical modes, depends on the aspect ratios given by the ratios of $k$, $n$, and $c$, where the last dictates an approximate wall-normal scale associated with the wall-normal distance to the critical layer. Consequently, the cross-stream component is weak for the VLSMs.

Of particular interest are the distributions of the wall-parallel components of the mode. Near the wall, in the region that gives the mode its "attached" designation, the phase of the mode monotonically decreases, leading to the aforementioned small angle of inclination to the wall. However, further from the wall the amplitude is smaller, but there is essentially zero phase variation in the wallnormal direction. Not only does this behavior mirror the expected phase variation beyond the $\pi$ phase jump associated with classical critical layer theory, ${ }^{36}$ but it is also in good agreement with cross-correlation results in the literature that had remained somewhat puzzling. ${ }^{52-54}$ Guala, Metzger, and McKeon ${ }^{54}$ observed similar shapes in the two-point cross-correlations, $R_{x x}\left(r_{x}^{+}, y^{+}, y_{\text {ref }}^{+}\right)$, in the near-thermally-neutral atmospheric surface layer, with temporal records transformed to the spatial domain using Taylor's hypothesis. The expected near-wall structural angle was observed with a reference location, $y_{r e f}^{+}$, close to the wall, while a sufficiently large $y_{r e f}^{+}$led to effective capture of a region of weaker correlation with reduced phase variation far from the wall. Interestingly, Chung and $\mathrm{McKeon}^{52}$ found similar behavior of the velocity field in LES of long channels conditioned on the occurrence of large-scale negative and positive velocity excursions, with the same dependence on the wall-normal location of the condition. Note that while there is some ambiguity concerning the interpretation of streamwise scale from both the cross-correlation results and conditional average procedures, it is clear that the dominant scales are of the order of several boundary layer thicknesses 
long in the streamwise direction. It also seems that the general variation of phase in the wall-normal direction described above is a common feature that can be more easily isolated by these techniques as the Reynolds number increases and the peak amplitude of the LSM/VLSM both increases and occurs further from the smaller-scale near-wall activity. We note also that the mode shown in Figure 9 has a non-negligible amplitude over at least 30\% of the radius, in which distance the change in mean velocity is approximately $80 \%$ of the centerline velocity, underscoring the underlying reason for the failure of Taylor's hypothesis for very large streamwise scales. ${ }^{43}$

For $K=(1,10,2 / 3)$, a simple prediction of the scaling of the peak location in streamwise amplitude, $y_{c}^{+}$, can be made, where $y_{c}^{+}$is understood to track with reasonable fidelity the wallnormal location of the critical layer. McKeon and Sharma ${ }^{1}$ showed that the wall-normal location where the response mode with $(k, n)=(1,10)$ first attains attached critical status occurs at $c=2 / 3$, independent of Reynolds number, and that this location lies within the region of logarithmic scaling of the mean velocity. The latter observation allows expression of the $y_{c}^{+}$in terms of the local mean velocity at the critical layer, two-thirds of the centerline velocity, solely by taking advantage of the log law and Reynolds similarity of the outer flow:

$$
\frac{1}{\kappa} \ln y_{c}^{+}+B=\frac{2}{3}\left(\frac{1}{\kappa} \ln R^{+}+B+C\right) .
$$

Here $\kappa$ is the von Kármán constant, and $B$ and $C$ are the additive constants associated with the mean velocity in the log region and the constant wake function. Solution of Eq. (16) with standard values for the constants ${ }^{1}$ leads to the prediction of

$$
y_{c}^{+}=0.8 R^{+2 / 3},
$$

which appears to be well-borne out by examination of the experimental data of Morrison et al. ${ }^{28}$ (Figure 13 of McKeon and Sharma ${ }^{1}$ ).

Despite the difficulties applying critical layer analysis to turbulent pipe flow identified earlier, it is worth mentioning a related result that is at least encouraging. McKeon and Sharma ${ }^{1}$ observed that classical critical layer analysis yields a scaling with $R^{+2 / 3}$ for the critical layer in the upper branch solution for neutrally stable modes, along with the more recognizable $R^{+1 / 2}$ for the wall layer in both upper and lower branches. The critical layer in the lower branch scales with $R^{+1 / 5}$. That the exponent from the classical analysis is in agreement with the scaling of the VLSM energetic peak gives hope that the $c=2 / 3$ for the VLSM determined observationally from our analysis is a fundamental theoretical result. However, the comparison of the variation of the location of the VLSM peak in the boundary layer data of Guala, Metzger, and McKeon ${ }^{54}$ and (a proxy for it) from Mathis, Hutchins, and Marusic ${ }^{55}$ shown in Figure 16 of McKeon and Sharma ${ }^{1}$ with the pipe result of Eq. (17) gives a hint that this behavior (the branch choice) may not be independent of flow geometry.

\section{Hairpin vortices and structural organization}

Our discussion of self-organization of coherent structure and its relationship with energetic large scale motions afforded from resolvent analysis begins with reference to the classical structure of the critical layer described by Kelvin, which hints at the origins of hairpin vortices. Figure 10 shows a sketch of the periodic, closed streamline structure seen by an observer moving at the critical

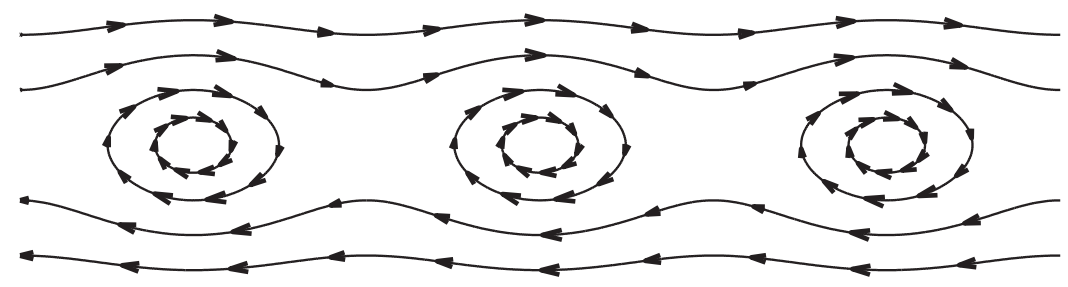

FIG. 10. Kelvin's cats' eyes (also called Kelvin-Stuart vortices): structure at the inviscid, laminar critical layer as observed by an observer moving at the critical velocity. 
velocity associated with a classical, inviscid (two-dimensional) critical layer. The three-dimensional wavenumbers expected from observations of wall turbulence (as opposed to the two-dimensional perturbations that emerge as the most unstable in linearly unstable laminar flow via Squire's theorem) suggest that any structure derived in the turbulent case will be more complex: at the very least, the superposition of left- and right-running velocity response modes suggests a three-dimensional vorticity variation, which we will show below appears to represent the commonly-drawn outline of a hairpin vortex.

A fundamental concern with considering coherent vortical structure in a turbulent field associated with a strong mean shear is the challenge of objective identification of rotation over shear. Recent reviews of this problem are given by, e.g., Jeong and Hussain ${ }^{56}$ and Chakraborty, Balachandar, and Adrian $;{ }^{57}$ for the simplified flow fields associated with the linear superposition of velocity response modes investigated here, all common identifiers give similar results, hence we choose to work with swirl, $\lambda$, which is given by the magnitude of the imaginary part of the complex conjugate eigenvalue pair associated with the velocity gradient tensor. This is, as are all the common diagnostics, a nonlinear function of velocity (a characteristic that leads rapidly to structural complexity, as we will show below). Therefore, while the velocity response modes can be linearly superposed to obtain approximations to the full velocity field of increasing veracity, a faithful representation of the full swirl field can only be obtained by summation of all active velocity response modes (with the correct amplitudes). However, our aim here is to show that the recognizable foundations of the structure observed in full flow can be traced to resolvent analysis, and specifically that phenomena such as evolving packets of hairpin vortices arise naturally from the resolvent analysis as studiable sub-units.

Figure 11 shows an isosurface of the swirl field associated with $K_{1}=(6,6,2 / 3)$, a critical mode in the terminology of Sec. II C that is just detached from the wall. Sharma and McKeon ${ }^{25}$ selected this response mode as one of a triadically consistent set that includes the VLSM (we summarize the full corresponding swirl field below). A periodic array of pro- and retro-grade hairpin-like vortices is found, where prograde implies rotation in the classical sense with a contribution to $-u v$, and the opposite for a retrograde vortex. This is as expected since the response modes represent the velocity distribution relative to the local mean velocity and there can be no mean contribution to the mean shear from the response mode. These coherent vortical structures can be understood phenomenologically

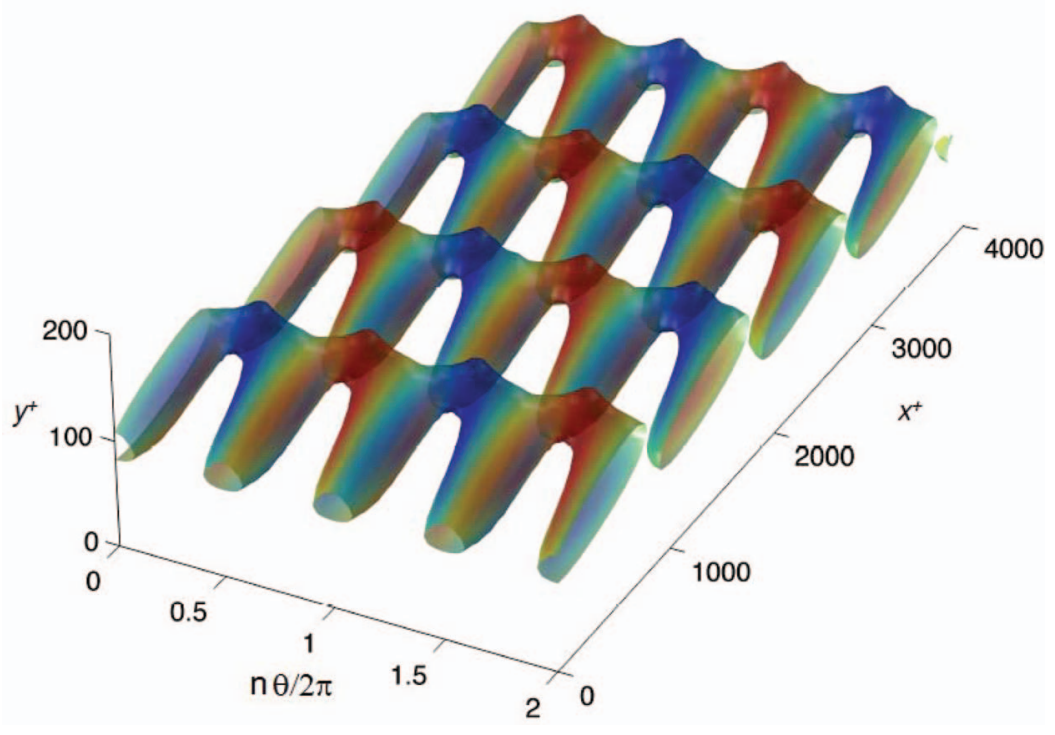

FIG. 11. The periodic array of pro- and retro-grade hairpin vortices associated with near-wall velocity response modes at $R^{+}$ $=1800$, identified by an isosurface of constant swirling strength at $50 \%$ of the absolute maximum value, color-coded with the local (model) azimuthal vorticity. Red and blue denote rotation in and counter to the sense of the classical (prograde) hairpin vortex, respectively. 
to be the three-dimensional equivalent of Kelvin's cats' eyes. Reference to Figure 7 confirms that the associated Reynolds stress is localized around the critical layer and detached from the wall, making the characteristics of this mode consistent with the foundational elements of Townsend's attached eddy hypothesis.

The swirl function identifies regions of rotational motion, but it is susceptible to contamination by local shear gradients in the sense that the absolute value of the swirl can be either increased or decreased by the simultaneous presence of shear. Most notably, the positive shear associated with the mean profile can be shown ${ }^{25}$ to increase (suppress) the swirl value associated with prograde (retrograde) hairpin vortex heads. This is a thresholding problem: rotational motion is still captured for both senses of vortex rotation, but the usual thresholding techniques applied to velocity fields lead to a systematic under-accounting of retrograde vortices. McKeon, Sharma, and Jacobi ${ }^{50}$ explored this phenomenon in the context of this work with respect to observations of swirl in the streamwise/wallnormal plane of a zero pressure gradient turbulent boundary layer, while Carlier and Stanislas ${ }^{58}$ documented earlier the general characteristics of the distributions of pro- and retro-grade hairpin vortices.

In the context of exploiting the resolvent analysis to demonstrate recognizable structure, we explore further the effect of local shear associated with combinations of velocity response modes. An energetic VLSM, for example, can be viewed as enforcing a periodic, enhanced and reduced local Reynolds number on the smaller motions, accompanied by positive and negative shear, $\partial u_{K} / \partial y$. In this sense, it can be considered to be an inactive motion in the terminology of Townsend. A similar effect occurs in the other velocity components and gradient directions, but the strongest of these is the wall-normal gradient of streamwise velocity, at least for the large scales which have long been associated with vortex organization in the literature. Thus the distribution of hairpin vortices with a particular $K$, which is periodic in the absence of other modes, will be modified by local shear associated with the linear superposition of any other (sufficiently energetic) velocity modes, and this modification gives rise to the self-organization of vortical structures familiar in the literature.

This phenomenon is described at length in Sharma and McKeon, ${ }^{25}$ but we demonstrate the complexity of vortical structure that can arise under the superposition of three modes in Figure 12. The three modes $K_{1}=(6,6,2 / 3), K_{2}=(1,6,2 / 3)(\mathrm{VLSM})$, and $K_{3}=(7,12,2 / 3)$ are triadically

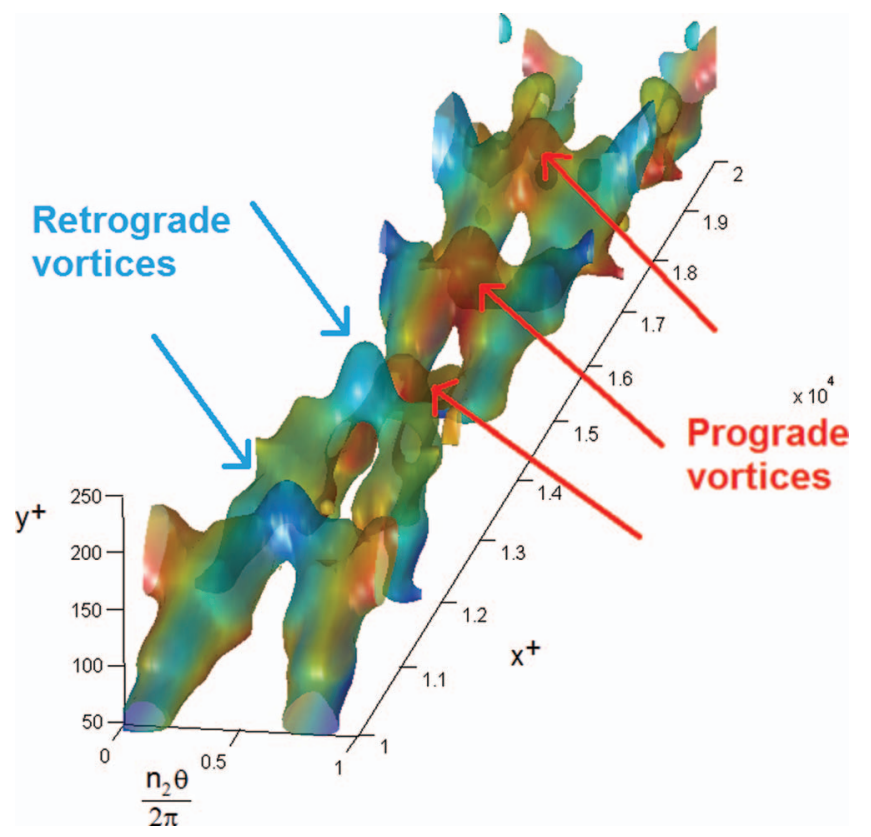

FIG. 12. Isosurfaces of constant swirling strength (33\% of maximum value) for the "ideal packet" at $R^{+}=1800$, color-coded by the local (model) azimuthal vorticity. Red and blue denote rotation in and counter to the sense of the classical hairpin vortex or prograde and retrograde vortices, respectively. 
consistent in $K$ and overlapping in the $y$ direction, and will be phase-locked in the sense that the identical wavespeed means that there is no relative motion between the modes and therefore no evolution of the packet. Sharma and McKeon ${ }^{25}$ selected relative mode amplitudes consistent with experimental observations, specified a $\pi / 2$ phase difference between the large-scale envelope of the two shorter modes and the VLSM in the streamwise sense and aligned the peak streamwise intensities in the azimuth, in order to generate the complex swirl distribution shown in Figure 12, which is recognizably a packet of hairpin vortices. Three prograde vortices with two retrograde ones sitting downstream and above the main packet can be clearly identified, along with some weaker vortices of both senses below the packet. The prograde hairpin heads occur further from the wall with increasing streamwise direction and are collocated with the inclined interface between positive and negative streamwise velocity fluctuation imposed by the VLSM (the location of maximum shear), giving the packet its distinctive shape. We find the complexity of coherent structure arising from even the idealized arrangement of only three modes to be striking.

This assembly of modes was deemed an "ideal packet" by Sharma and McKeon ${ }^{25}$ because of its lack of evolution (matched wavespeeds) and spatial alignment. Note that this is developed as an example of structure from the resolvent analysis that can be simply interrogated rather than a structure that we expect to observe in a real flow. Relaxing any of the alignments or superposing more velocity response modes will disrupt the coherence of such a packet, such that isosurfaces of the swirl diagnostic identify the canes and arches generally observed in moderate Reynolds number wall turbulence. Permitting a range of wavespeeds leads to packet evolution in space and time. However, Sharma and $\mathrm{McKeon}^{25}$ speculate that this combination of modes is dynamically important and likely to be self-sustaining, in addition to being triadically compatible, and termed it a "turbulence kernel." It should be understood that the Reynolds stress associated with the correct assembly of velocity response modes supports the (currently assumed) mean velocity profile, but in the resolvent interpretation this comes from the mean contributions to the $-u v$ distribution rather than the concentration of stress associated with the hairpin heads, both of which can be observed in the bottom panes of Figure 7. The resolvent analysis offers a different explanation for the source of the Reynolds stress from that offered by the attached eddy hypothesis. In the latter, the distribution of eddies effectively describes the mean velocity profile and the scaling of the velocity fluctuations.

A simple superposition of first singular modes gives clear insight into a mechanism of selforganization in wall turbulence. In crude terms, the local variations of shear associated with the linear superposition of velocity modes bias the identification of structure to the edges of regions of energetic large scale streamwise motion (the zones of constant momentum of Adrian, Meinhart, and Tomkins ${ }^{59}$ ), implying that coherent vortex structure is most likely to be observed around streamwise streaks of low velocity, as shown, e.g., in the elegant conditional averaging of time-resolved particle image velocimetry (PIV) by Dennis and Nickels. ${ }^{60}$ Perhaps, then the well-known organizational characteristics of LSMs and VLSMs in terms of the observed preferential wall-normal and spanwise locations of hairpin vortices flanking large scale energetic motion can be at least partially attributed to the choice of nonlinear diagnostic for measuring swirling motions.

\section{Summary of results regarding unperturbed wall turbulence}

The agreement between response modes and observations of the real flow detailed above suggests that the low rank approximation to the NSE afforded by the first singular response modes over the range of wavenumbers and frequencies observed in real flows is a useful tool for modeling wall turbulence and illuminating dominant flow dynamics. It is important to stress that the dynamics of the flow are captured and explained by the analysis. More information on the organization of the flow, essentially the amplitudes and relative phases of the velocity response modes, appears to be available from consideration of the triadic interactions such as those presented by our turbulence kernel; this is beyond the scope of the current manuscript, but is explored in Sharma and McKeon. ${ }^{25}$

Another benefit of using the systems paradigm is the potential for carrying over closed-loop control thinking and technology to explore the possibilities for turbulence control in the presence of an almost ideal model. We explore strategies for control in Secs. IV A-IV D. 


\section{EXPERIMENTAL MANIPULATION OF THE TURBULENT BOUNDARY LAYER USING DYNAMIC ROUGHNESS ACTUATION}

Bewley ${ }^{61}$ observed that "coordination of simulation-based modeling and control design is largely an unsolved problem." The key difficulty is that, by design, the dynamics of the uncontrolled system are often quite different from the controlled. This makes an experimental demonstration showing that it is possible to use the approach to provide a deterministic change to wall turbulence essential to evaluating the use of the resolvent framework in modeling forced-flow dynamics.

A strategy for incorporating what is, in essence, open-loop control via our analysis can be identified in Figure 3, where external forcing is used to over-stimulate a single $K$ component of the linear system. The low rank nature of the resolvent suggests that the exact radial form of the forcing is not essential so long as there is a component in the most amplified direction $\left(\phi_{K, 1}\right)$, since this is what dominates the response. In the context of the fully coupled Navier-Stokes system, this is conceptually analogous to adding a tracer to the system, highlighting the energy transfer that takes place as a consequence of nudging the turbulence away from its natural self-consistent state, and thus is interesting in its own right as well as providing proof that the effect of the external forcing can be predicted through the analysis described above. Harnessing the sensitivity of flow itself to individual forcing inputs provides an optimal method of "tickling" the flow.

The practical attraction of wall-based actuation for real-world applications is strong, although this will necessarily lead to non-optimality of the forcing associated with imperfect coupling from the wall to the body of the flow (discussed further below). Many methods of actuating the flow from the wall exist, as reviewed recently by Cattafesta and Sheplak, ${ }^{62}$ however the need to isolate individual $K$ modes in a continuous, practically implementable surface suggested the use of a dynamic roughness: distributed surface roughness elements with a temporally varying amplitude. Consider the "active eggbox" wall shown in Figure 13, a geometry with single wavenumbers in the streamwise and spanwise directions. When actuated at a single frequency, $\omega$, it provides a harmonic temporal variation in amplitude. Such a surface is capable of coupling directly with the flow in a way consistent with the resolvent analysis, under the assumption that the downstreamtraveling component of the upstream- and downstream-traveling wave combination that is linearly superposed to give the standing wave at the wall is preferentially amplified. This is consistent with our understanding of the characteristics of wall turbulence and the singular values obtained by McKeon and Sharma. ${ }^{1}$ An initial demonstration of such coupling was provided by McKeon, ${ }^{63}$ who enforced a linearized equivalent boundary condition (effectively wall transpiration) in direct numerical simulation (DNS) of fully developed turbulent channel flow to show that the resulting disturbance shape could be predicted using only the mean velocity profile as input.

While conceptually simple, the practical implementation of dynamic roughness requires some careful design, although advances in the field of smart materials offer hope for future developments of large-area morphing surfaces. In this section, we review the results of Jacobi and McKeon ${ }^{24}$ who employed a simplified version of a fully developed morphing surface over a limited wall area in

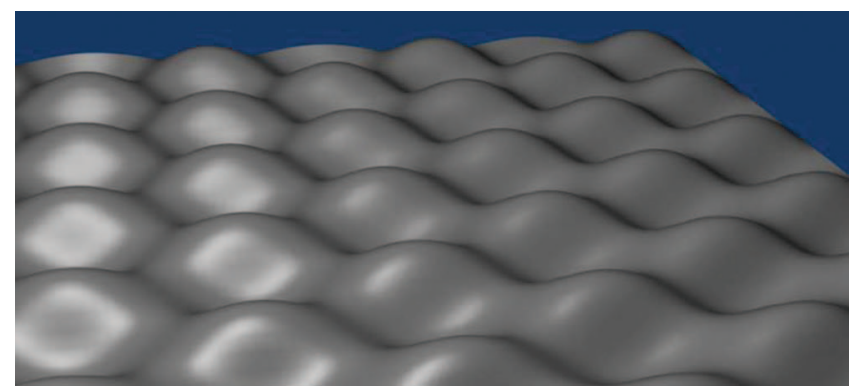

FIG. 13. An example of a morphing surface geometry capable of simple interaction with the resolvent analysis: an "active eggbox" that introduces a single $K$. A roughness geometry described by single wavenumbers in the wall-parallel directions $(k, n)$ is actuated at a single frequency, $\omega$, leading to harmonic temporal variation in amplitude. 
order to produce forcing amenable to the resolvent analysis described above, adapted for the zero pressure gradient turbulent boundary layer.

\section{A. Turbulent boundary layer excitation using a dynamic roughness impulse}

The experiments of Jacobi and McKeon ${ }^{24,64}$ employed a simple top-hat (two-dimensional) spatial impulse of dynamic roughness as a proxy for a fully developed morphing surface. In practice this is an array of small spanwise ribs with amplitude varied harmonically in time. This geometry allows significant experimental simplifications, while affording a unique perspective by leveraging a substantial knowledge base in wall-bounded flows that have been knocked out of equilibrium by deterministic perturbations. The literature on turbulent boundary layers under general perturbation by spatially impulsive changes in surface geometry, roughness, pressure gradient, and other factors, as well as fully developed flow over two-dimensional roughness elements is reviewed in Jacobi and McKeon $^{24,64}$ and will not be repeated here in the interests of brevity. We stress, however, that the previous studies considered static perturbations as opposed to the dynamic amplitude variation that will be described below.

The essential details of the experimental study follow (full details of the facility, operating conditions and measurement techniques can be found in Jacobi and $\mathrm{McKeon}^{24,64}$ ). The experiments were performed in a low free-stream turbulence, incompressible, zero-pressure-gradient turbulent boundary layer. The basic roughness geometry for these experiments is shown in Figure 14. The roughness impulse consists of four spanwise bars of $k$-type roughness, each $1.57 \mathrm{~mm}$ thick, $1 \mathrm{~mm}$ tall (above the surface of the wall, in the case of the static roughness perturbation experiment), and separated (interstitially) by $6.35 \mathrm{~mm}$, illustrated in Figure 14. The entire patch extends for $25.3 \mathrm{~mm}$ in the streamwise direction, or approximately 1.5 times the mean boundary layer thickness $\left(\delta_{99}\right)$ of the unperturbed boundary layer, denoted $\delta_{0}$. The flat plate itself was modified to allow the roughness elements to pass through slots, in order to allow the roughness to be mechanically actuated by a dc motor with a piston and crank-shaft assembly mounted outside the wind tunnel and operating at a fixed frequency of $30 \mathrm{~Hz}$. An almost perfect sinusoidal variation of amplitude from flush with the surface to peak amplitude of approximate height $1.64 \mathrm{~mm}$ and root mean square (rms) of 1.16 $\mathrm{mm}$ is obtained, as determined by a linear encoder which is also utilized to phase-lock velocity measurements to the position of the oscillating roughness. The peak oscillating amplitude is fixed in order to maintain an approximate match between the rms amplitude of the dynamic perturbation and the constant amplitude of the static roughness elements, which were studied in companion experiments.

The selection of wavenumber/frequency combination, $K$, for study was based on spectral considerations described below and on the practicality of implementing the perturbation. The spanwise wavenumber, $n$, is fixed by the spanwise uniformity of two-dimensional roughness. PIV measurements confirm the two-dimensionality of the downstream flow. The streamwise wavenumber $k$

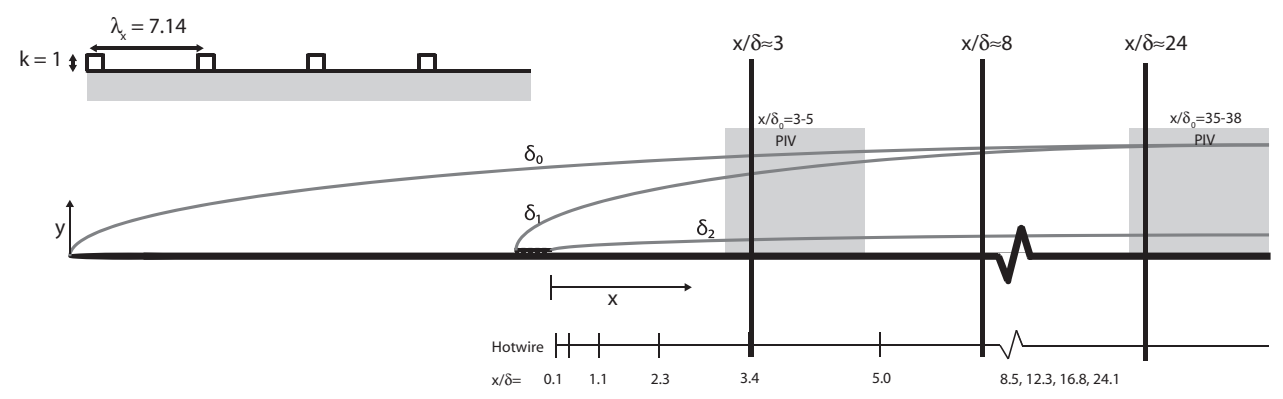

FIG. 14. A schematic of the arrangement of the flat plate, the roughness strip, and the diagnostic locations; not to scale. The internal layers are also marked in order to provide an idea of their relative sizes and development rates. The first internal layer, $\delta_{1}$ grows rapidly; the second, $\delta_{2}$, grows slowly. The mean boundary layer thickness $\left(\delta_{99}\right)$ at the location of the roughness in the unperturbed flow is denoted $\delta_{0}$. The three key measurement locations highlighted in the subsequent discussion are marked with black lines. 
is a fixed function of the roughness impulse and flow parameters; the roughness geometry described above leads to a streamwise wavenumber of $\lambda_{x} \approx 18.70$ or $k=0.336$. Note that nondimensionalization for the boundary layer results is performed using the boundary layer thickness just upstream of the roughness impulse, $\delta_{0}$ in Figure 14, and the freestream velocity. The wavespeed is then assigned by the rotation frequency of the DC motor, which was picked to target the lower end of the range of naturally-occurring frequencies in the boundary layer. The resulting convection velocity coefficient is $0.464-0.066 i$ (where the imaginary component is due to the decay of physical disturbances), which places the critical layer at $y^{+} \approx 0.04$ for the unperturbed velocity profile at the first streamwise measurement location. Thus the actuation introduces a perturbation with $K$ reminiscent of a synthetic, two-dimensional very large scale motion.

This assignment of wavenumbers can be performed only because of the strength of a roughnesstype impulse on the flow. Roughness type perturbations are much stronger than those produced by the vibrating ribbons used in early transition experiments like that of Schubauer and Skramstad, ${ }^{65}$ as well as the comprehensive study on perturbation of the turbulent boundary layer performed by Hussain and Reynolds. ${ }^{66-68}$ In fact, the roughness-type impulse was used in Jacobi and McKeon ${ }^{64}$ precisely because it serves so effectively to disrupt the equilibrium condition of the turbulent boundary layer. Moreover, the simple geometry of two-dimensional roughness elements provides experimental simplification and, importantly, more control over the perturbation wavenumber set $(k, n, \omega)$. While the non-equilibrium flow makes reconciliation of the experimental results with the theory more challenging, as described below, it still provides a useful demonstration of the approach.

In what follows, we present a selection of point and field measurements of the flow field made downstream of the roughness at streamwise locations using single normal hot-wires and streamwise/wall-normal aligned PIV. The streamwise stations are marked in Figure 14 and correspond to Reynolds numbers in the unperturbed flow between $R e_{\theta}=2560-4070$ or $\delta^{+}=910-1200$ (note the difficulty of making even an empirical determination of the friction velocity in a boundary layer out of equilibrium).

\section{B. Non-ideal forcing: Separation of roughness effects and dynamic forcing}

Jacobi and McKeon ${ }^{64}$ characterized the boundary layer downstream of the roughness geometry described above with a constant (time-independent) amplitude matched to the rms amplitude for the dynamic case in order to provide a baseline roughness perturbation condition. Of course the dynamic and static effects are unlikely to be able to be linearly superposed, given the spatial inhomogeneity of the change in boundary condition. Note however, that in the case of fully developed, spatially periodic roughness, a static perturbation corresponds conceptually to a spatially varying mean flow and the excitation of a spread of temporal frequencies associated with local separation and flow variation around the roughness elements. We briefly review the key features associated with the statically perturbed boundary layer in order to introduce the reader to the influence of this undesired effect with respect to the dynamic results that follow.

The static impulse of roughness produces a significant modification of the mean flow field, observed in the mean velocity profile, higher order statistical moments of the velocity fluctuations, and structural features of the boundary layer, including the distribution of vorticity. Certain phenomena are important very near the roughness (for both the static and dynamic perturbations), including a complex recirculation zone immediately downstream of the last roughness element and the possibility of the addition of coherent structure associated with vortex shedding over the elements, etc. The former effect is avoided by taking data sufficiently far downstream, while any effect associated with the latter is not sufficiently strong to be distinguishable from the outward displacement of incoming near-wall turbulence activity due to the presence of the perturbation. In addition, the impulse of roughness generates the two internal layers within the boundary layer marked in Figure 14. Internal layers are produced at the transition between boundary conditions, from a smooth to rough wall and again from a rough to smooth wall, and represent the extent to which the new boundary condition has influenced the nearby flow. Only when the internal layers have grown to the edge of the boundary layer can the flow be said to approach the equilibrium corresponding to an unperturbed flow; this growth is known to be slow (consistent with the cartoon of Figure 14) such that the return to 
equilibrium takes tens of boundary layer thicknesses, with the exact value dictated by the strength of the roughness perturbation.

One important effect identified by Jacobi and McKeon ${ }^{64}$ is the region of elevated mean shear in the perturbed case (relative to the unperturbed mean velocity profile) that occurs in the region between the internal layers, deemed a stress bore after the study (albeit of a different non-equilibrium flow) by Smits, Young, and Bradshaw. ${ }^{69}$ Not only does the stress bore manifest itself in surplus mean shear between the two internal layers, but it also produces an increase in mean turbulence intensities in precisely the same region. A velocity scale based on this modified local shear can be shown to collapse the streamwise turbulence intensities downstream of the roughness. In summary, the return of the flow to equilibrium following the transitions in wall boundary condition occurs over a significant downstream distance and is strongly related to the existence of the stress bore.

When the roughness was actuated dynamically, nearly all of the same features of the stress bore were observed, along with additional flow features associated specifically with the periodic oscillation. The combination of these two effects-the stress bore associated with the roughness impulse itself and the periodic oscillation introduced into the flow by the mechanical actuationmakes separating the periodic effect difficult when examining the turbulence statistics. However, employing a spectral perspective provides significant insight into the effects of both static and dynamic perturbations; the statistical and spectral signatures of these non-ideal effects are identified in the spectral results shown below.

The premultiplied, composite spectrum of the streamwise velocity fluctuation is shown in Figure 15 for the unperturbed flow at the furthest downstream measurement location (or, equivalently, the highest Reynolds number) and for the perturbed flow downstream of the static and dynamic impulses at the three measurement locations identified in Figure 14. Taylor's hypothesis has been employed to convert from the frequency domain to the wavelength domain using the local mean velocity, for reasons of convention. Under both static and dynamic perturbation, incoming near-wall turbulence activity is displaced away from the wall by the two-dimensional roughness, resulting in a broadband increase in turbulence energy away from the wall contained within the stress bore identified earlier. Moreover, downstream of the perturbation, the signature of the near-wall cycle is less strong, indicating that the region within the second internal layer can be likened to a newly established boundary layer over the smooth wall condition downstream of the impulse. In addition to the displacement effect associated with the stress bore, the flow under dynamic perturbation shows a coherent signature of the input forcing frequency across most of the boundary layer to downstream distances in excess of $20 \delta$. Besides the fundamental frequency of the forcing, a number of harmonics were also observed, which decayed rapidly with increasing streamwise distance and as such are not considered in detail here.

The persistence of the linear response of the fundamental frequency far downstream suggests a successful coupling between the forcing and an innate mode of the underlying base flow. Phaselocked velocity measurements provide a natural means by which this particular mode (frequency) within the turbulent boundary layer can be isolated and visualized; we use this technique to identify both linear and nonlinear aspects of the response of the boundary layer to the dynamic forcing.

\section{Identification of the dynamic perturbation through phase-locking}

The strong signature across the boundary layer at the forcing frequency for a considerable downstream distance indicates that the excited perturbation is strong and coherent, and that it decays relatively slowly. It also makes the recovery of its characteristics possible via phase-locking of the instantaneous velocity signal from the hotwire or PIV to the encoder measurement of the vertical position of the roughness. Adding an applied harmonic forcing $\breve{\mathbf{f}}_{k}$ of amplitude $\epsilon$ to Eq. (7) gives

$$
\mathbf{u}_{K}+\epsilon \breve{\mathbf{u}}_{K}=\mathcal{H}_{K}\left(\mathbf{f}_{K}+\epsilon \breve{\mathbf{f}}_{K}\right),
$$

where, due to the linearity of the resolvent, we see that the first order effect should be proportional and at the same wavenumber. Of course, the mean velocity profile will adjust and therefore affect the resolvent. For now, we identify this effect through experimental measurements. 

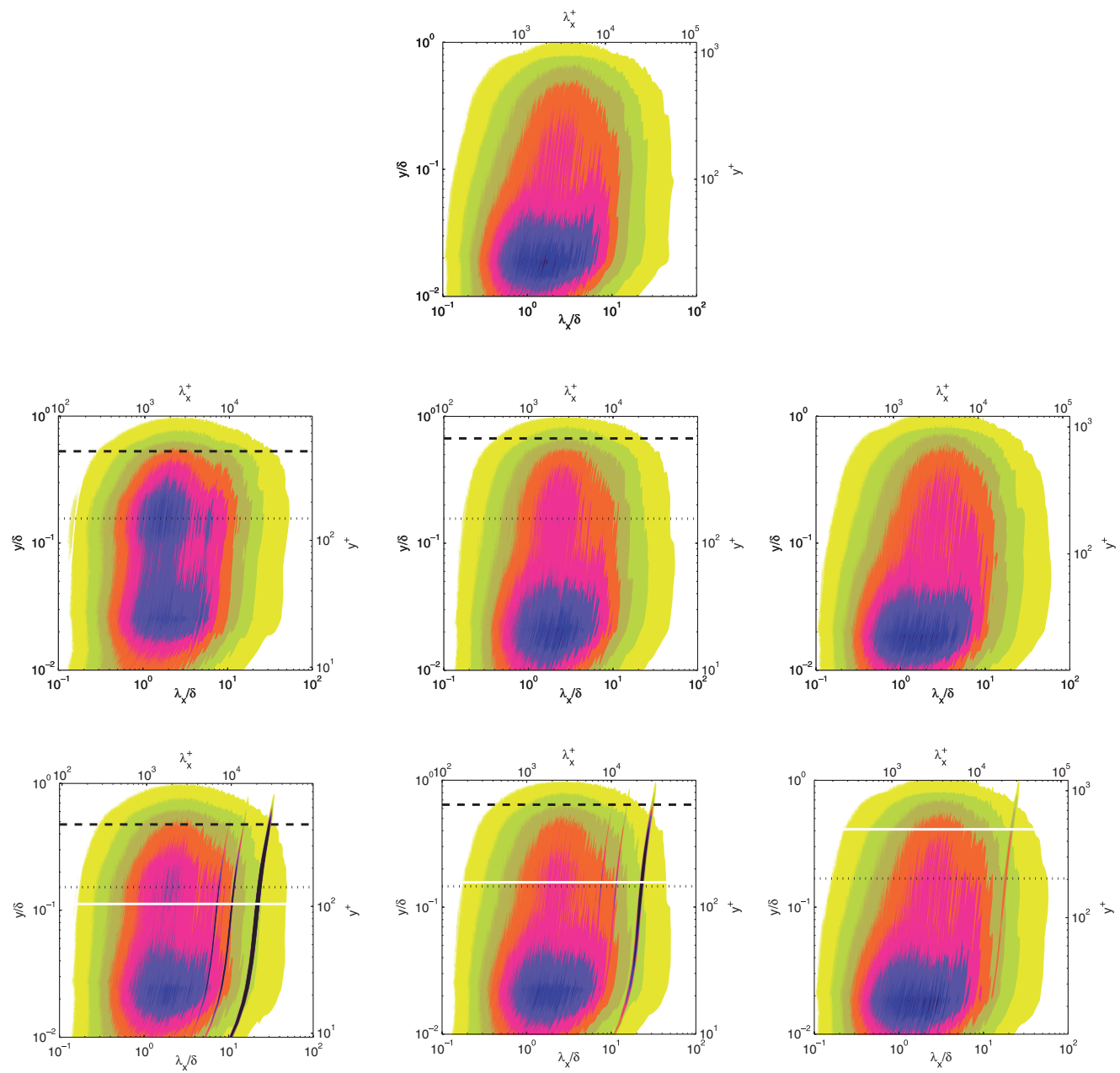

FIG. 15. Composite spectra for the unperturbed and perturbed flows. Ten contour levels, equally spaced across the color bar, are indicated. Top row: unperturbed case, measured at $R e_{\theta}=4040$. Middle row: static roughness perturbation; bottom row: dynamic perturbation. For the latter two rows, left to right is the direction of increasing streamwise distance downstream from the impulse, at approximately $x / \delta=3,8,24$. The mean internal layer locations, when distinguishable, are denoted: - - first internal layer, and $\cdots$ second internal layer, following Figure 14. The solid white lines in the dynamically perturbed case indicate the location of the (local) critical layer.

The instantaneous velocity field that results from the addition of the applied harmonic forcing can be decomposed into a component associated with the external forcing itself, $\breve{\mathbf{u}}_{K}$, the velocity associated with the natural forcing (i.e., the natural nonlinearities in the flow), $\mathbf{u}$, and the underlying time-averaged velocity field, $\mathbf{u}_{0}$. This type of phase-locked decomposition was first proposed by Hussain and Reynolds ${ }^{66}$ and was employed in Jacobi and McKeon $^{24}$ using different notation, where the time-averaged field was represented by $U$, the phase-locked (periodic) velocity component associated with forcing at $K$ was denoted $u_{K}$, and the turbulent fluctuations about the periodic component as $u^{\prime}$. The fluctuating component (denoted $u^{\prime}$ in the earlier studies, now $\mathbf{u}$ to be consistent with the theoretical discussion) then includes the motions of all wavenumbers in the forced flow field besides the external forcing itself. These two equivalent forms of the phase-locked decomposition of the externally forced velocity field are shown in Eq. (19),

$$
\begin{aligned}
\tilde{u_{i}} & =\left(U+u_{K}+u^{\prime}\right)_{i}, \\
\tilde{\mathbf{u}} & =\left(\mathbf{u}_{0}+\breve{\mathbf{u}}_{K}+\mathbf{u}\right) .
\end{aligned}
$$



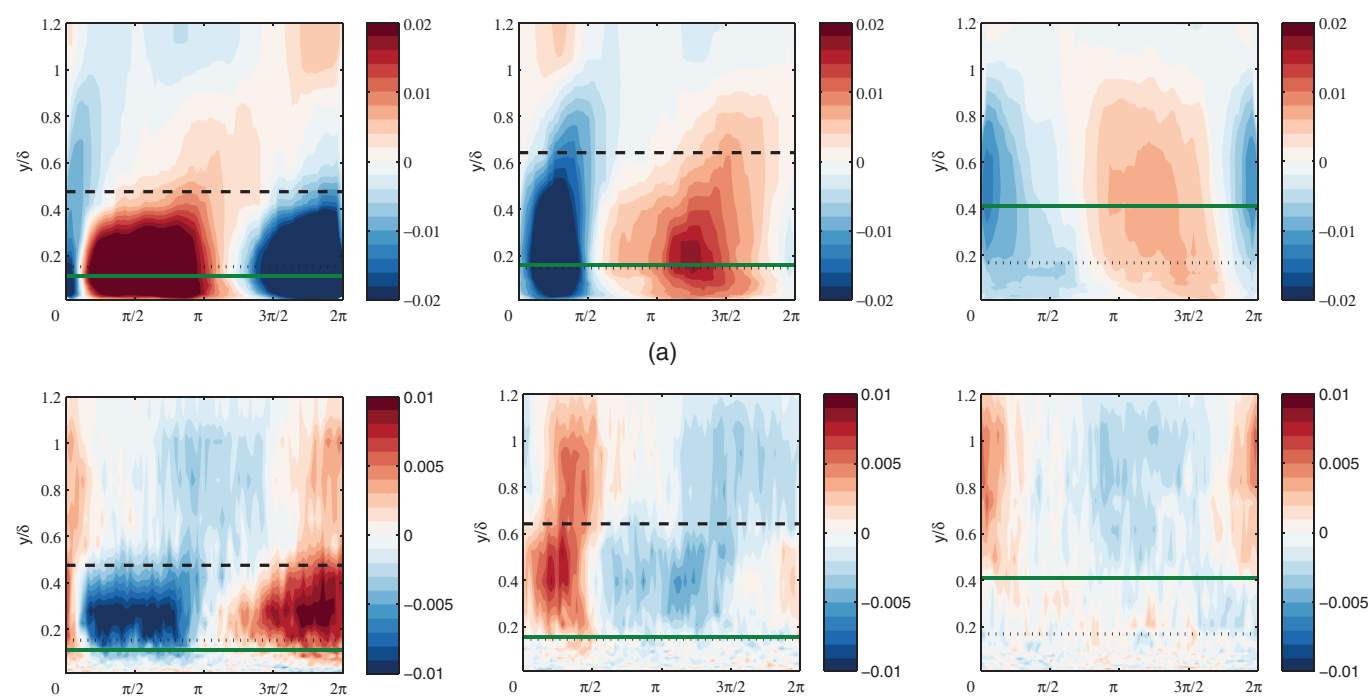

(b)

FIG. 16. Phase-locked maps of the streamwise component of the velocity field measured from the hotwire over an average period (abscissa $t \in[0,2 \pi]$ ) in outer units (ordinate $y / \delta$ ) at the three streamwise locations indicated in Figure 14 . (a) The periodic component, $\breve{u}_{K}$. (b) The rms of the fluctuating component, $u$, with the mean value subtracted. The mean internal layer locations, when distinguishable, are denoted: - - first internal layer, and $\cdots$ second internal layer. The solid green lines indicate the location of the critical layer, ascertained from the maximum amplitude of the streamwise velocity mode.

Phase-locked measurements at different streamwise locations also allow determination of the precise streamwise wavenumber, $k$, corresponding to the periodic (but decaying) velocity component, namely $k=0.336+0.048 i^{24}$

The downstream variations of a mean period of the streamwise and wall-normal velocity components, $\breve{u}_{K}$ and $\breve{v}_{K}$, are shown in Figures 16(a) and 17(a), respectively. The locations of the internal layers and the critical layer corresponding to the input conditions are identified by the horizontal lines. For both velocity components, the mode shapes are well defined, with distinctive variations across the boundary layer. The streamwise component mode shape shows a very shallow downstream inclination (rapid phase variation) near the wall, which transforms into an upstream inclination in the region past the second internal layer. Far downstream, the streamwise component mode shape appears to be uniformly inclined in the downstream direction. The (weaker) wall-normal component mode shape shows very little phase variation across the boundary layer. The critical layer location tends to occur in close proximity to the second internal layer, and grows away from the wall more quickly than the internal layer, thus moving further into the region of the stress bore with increasing downstream distance. Near the location of the critical layer, the phase of the streamwise component mode shape appears to be constant for a noticeable wall-normal extent, in between the regions of downstream inclination near the wall, and upstream inclination near the first internal layer.

Interestingly, the footprint of the forcing also manifests itself in a periodic variation of the amplitudes of turbulent fluctuations at other scales, as shown with the time-mean removed in Figures 16(b) and 17(b). The phase-locked variation of the rms of the fluctuations- $u$ and $v-$ display distinctive and well-defined mode shapes that are correlated with the periodic signals, $\breve{u}_{K}$ and $\breve{v}_{K}$ in a manner that varies in a non-trivial way with wall-normal location.

The persistence of the well-defined large scale mode at the input frequency confirms the dominant linear effect predicted by the resolvent analysis, while its imprint on the fluctuating components can only be due to nonlinear effects, either associated with the imperfect nature of the input forcing (with respect to the single $K$, shape of $\breve{\mathbf{f}}_{K}$ - less likely, or the coupling from the wall to the flow) or spectral organization induced by the excited large scale. A similar imprint of the energetic very large scale motions on small-scale turbulence in unperturbed boundary layers was identified by Bandyopadhyay and Hussain ${ }^{70}$ and further developed in the context of a proposed amplitude- 


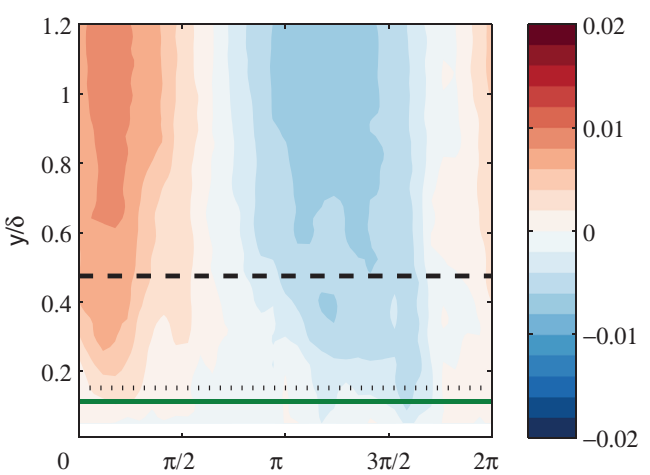

(a)

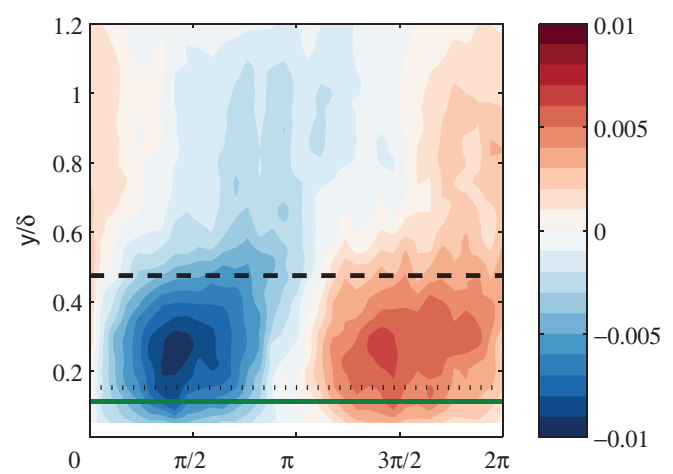

(b)

FIG. 17. Phase-locked maps of the wall-normal component of the velocity field measured from the PIV over an average period (abscissa $t \in[0,2 \pi]$ ) in outer units (ordinate $y / \delta$ ) at the first downstream location indicated in Figure $14, x / \delta \approx 4$. (a) The periodic component, $\breve{v}_{K}$, and (b) the rms of the fluctuating component, $v$, with the mean value subtracted. The mean internal layer locations, when distinguishable, are denoted: - - first internal layer, and $\cdots$ second internal layer. The solid green lines indicate the location of the critical layer, as measured from the hotwire measurements at the equivalent streamwise location.

modulating effect of large-scale motions on the envelope of small-scale motions by Mathis, Hutchins, and Marusic. ${ }^{55}$ Those analyses isolate the large-scale component from the instantaneous streamwise velocity signal (by low-pass filtering) and correlate it with an envelope of the remaining small-scale component, revealing a wall-normal variation of the cross-correlation that has been interpreted as an apparent amplitude modulation. Chung and $\mathrm{McKeon}^{52}$ observed that this trend in the correlation between the two signals can be more naturally considered in terms of a phase shift, where they are in-phase near the wall where the correlation is high and positive, and half a cycle out-of-phase (anti-correlated) away from the wall, where the correlation is high and negative. Recent work ${ }^{25}$ suggests that the location of the $\pi / 2$ phase shift corresponds to the location of the critical layer associated with the VLSM described in Sec. III B.

Our investigations into the apparent amplitude-modulation effect in order to consider the relationship between large- and small-scale motions in terms of the relative phase between them in both unperturbed and perturbed flows are ongoing, ${ }^{25,71}$ but the results lie outside the scope of this review. However, for the purposes of expanding on the claim made earlier that the actuation input excites a synthetic very large scale motion, we note that the phase description of the large-to-small scale interaction in the perturbed case has distinct similarities with the equivalent characterization of the unperturbed flow, but with modifications concentrated on the regions where dynamic forcing and roughness effects are expected. In particular, a similar phase variation is shown with respect to the location of the critical layer of the input perturbation rather than the unperturbed VLSM, as can be qualitatively observed in Figure 16. Note that correlating $\breve{u}_{K}$ with the envelope of $u$ is approximately equivalent to the more usual correlation of a Fourier-filtered large scale with the envelope of small-scale activity in this case because the forcing corresponds to the very smallest wavenumbers that contain non-negligible energy in the boundary layer.

\section{Predicting the synthetic large-scale motion}

The foregoing phase-locked analysis of the experimental data provides qualitative encouragement that a mode innate to the turbulence can be excited by the dynamic roughness. However a quantitative implementation of the resolvent analysis requires treatment of the semi-infinite (rather than closed) domain associated with the turbulent boundary layer, the streamwise inhomogeneity (non-parallel nature) of the flow, and the finite spatial extent of the original impulse along with the non-equilibrium effects of the stress bore (discussed above). Nevertheless, the resolvent analysis introduced in McKeon and Sharma ${ }^{1}$ can be applied to the boundary layer flow with the following straightforward modifications. 
The NSE under the boundary layer approximation can be rewritten using streamwise/wallnormal streamfunctions, $\zeta(x, y, t)_{k}$, as the divergenceless basis under the assumption of a twodimensional flow response, justified by the spanwise uniformity of the roughness impulse and the resulting downstream flow. Additional assumptions are required concerning locally parallel flow and a fully-developed response of the boundary layer to impulsive forcing in order to justify the use of Fourier modes as the optimal bases in the wall-parallel and temporal directions. Then the streamfunction can be written in terms of propagating waves with amplitude $\varphi(y)_{K}$, as shown in Eq. (20),

$$
\zeta(x, y, t)_{K}=\varphi(y)_{K} e^{i K \cdot \mathbf{x}}
$$

leading to the resolvent formulation of Eq. (21), where $\hat{\mathcal{H}}_{K}$ denotes the resolvent relating forcing and streamfunction

$$
\varphi(y)_{K}=\hat{\mathcal{H}}_{K}\left(\mathbf{f}_{K}+\breve{\mathbf{f}}_{K}\right)
$$

As described above, the precise wavenumber triplet can be obtained in a straightforward manner, but the choice of mean velocity profile to use in the resolvent formulation presents a significant difficulty. Using the perturbed velocity profile tends to better predict the mode shapes in a general sense, as would be expected since the perturbed profile reflects the mean effect of the stress bore. However, the difficulty in extending the perturbed profile to the wall (necessary for the computational domain) was judged ${ }^{24}$ to necessitate use of the unperturbed velocity profile in order to have confidence that the predictions were the result of experimental observations and not a numerical artifact from connecting an experimental profile to the wall (another issue associated with the non-equilibrium flow resulting from the use of a spatial impulse of roughness). As will be shown below, a direct consequence of the unperturbed profiles failing to capture the effect of the stress bore is that the predictions of the velocity response mode shapes tend to be most deficient precisely in the region of the stress bore, between the mean edges of the two internal layers.

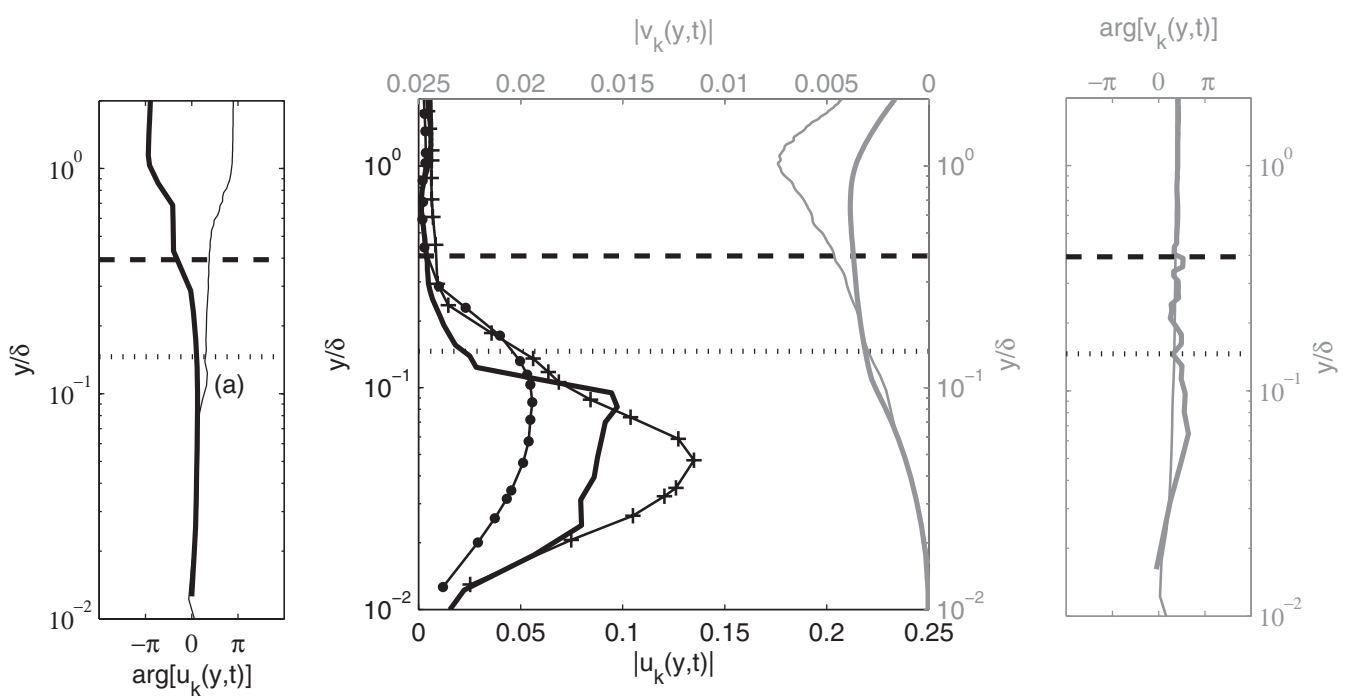

FIG. 18. The amplitude and phase of the velocity modes from the experiment and the resolvent analysis. The amplitude variation is shown in the middle pane. $\breve{u}_{K}(y, t)$ is shown on the lower-left axes: - from the resolvent analysis using the unperturbed velocity profile; - - - from the resolvent analysis using the perturbed velocity profile; $x / \delta=0.1+; 2.3 \times$ from the experimental hotwire measurements. $v_{K}(y, t)$ is shown in gray on the upper-right axes: - from the resolvent analysis using the unperturbed velocity profile; - from the experimental PIV measurement with PIV window centered at $x / \delta \approx 4$. The left pane indicates the phase of $\breve{u}_{K}(y, t)$, and the right pane indicates the phase of $\breve{v}_{K}(y, t)$. The (a) marks a distinctive variation in phase which, to our knowledge, is a robust feature of all Orr-Sommerfeld type solutions. The location of the internal layers have been marked for the streamwise component: --- the first internal layer; $\cdots$ the second internal layer. 

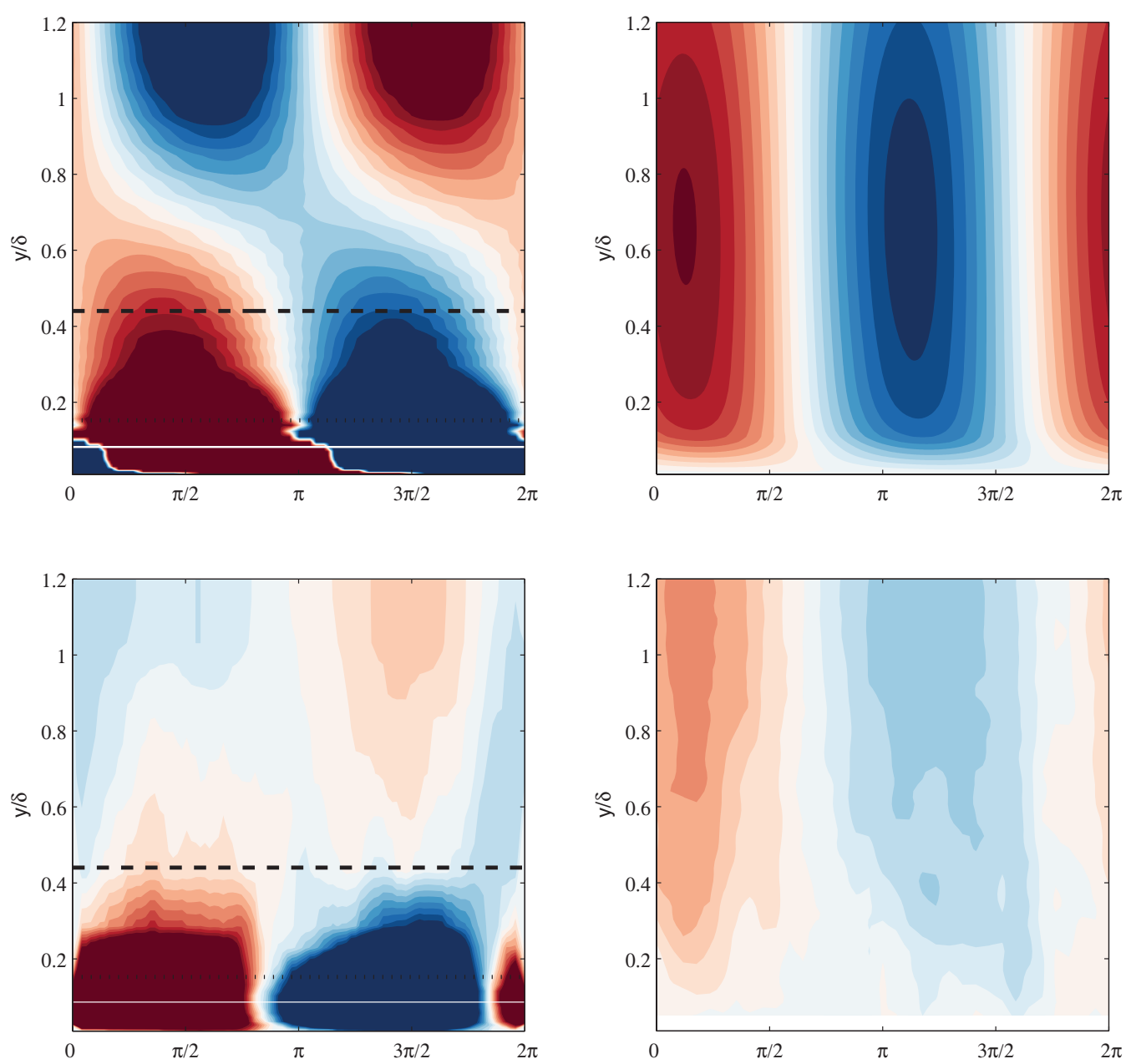

FIG. 19. Comparison of the resolvent calculations and phase-locked measurements. (Top) Maps of the calculated mostamplified singular mode over an average period $(t \in[0,2 \pi]) . \breve{u}_{K}(y, t)$ and $\breve{v}_{K}(y, t)$, left and right. (Bottom) The corresponding experimentally measured maps at $x / \delta \approx 2.3$, as in Figure 16(a).

The amplitude and phase profiles of the first singular mode of the resolvent operator formulated under the experimental conditions demonstrate encouraging agreement with the phase-locked velocity decomposition of the experimental data (Figure 18). The resolvent analysis captures the location of the peak amplitude in both the streamwise and wall-normal velocity modes, as well as the phase of the wall-normal mode. Within and even slightly beyond the second internal layer, the resolvent method provides a reasonably accurate prediction of the experimentally excited large scale motion. A noticeable divergence of the phase of the streamwise velocity occurs, however, in precisely the region between the two internal layers in which the stress bore distorts the mean shear. The effect of this distortion is easily seen in Figure 19, which shows the variation of predicted and observed streamwise and wall-normal velocity over a mean period. The predicted mode shapes tend to be inclined downstream consistently across the boundary layer, much like the experimental modes measured very far downstream from the perturbation, but the synthetic large scale observed nearer to the perturbation shows an upstream inclination far from the wall. In other words, the synthetic large scale appears warped by the presence of the stress bore, and this warping due to the flow non-equilibrium is not captured by the resolvent calculations.

A schematic of the shape of the excited streamwise velocity response, $\breve{u}_{K}$, including the location of the critical and internal layers is given in Figure 20. Although the method is significantly limited by the presence of the stress bore, which occurs as a direct consequence of the choice of actuation 


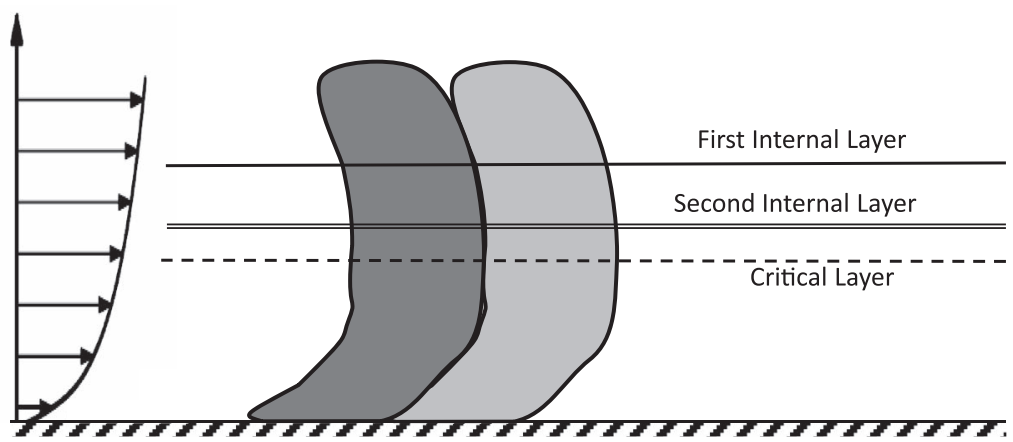

FIG. 20. A sketch of the physical form of the experimentally observed mode shape, emphasizing the distortion produced by the stress bore, as well as the location of the internal and critical layers. This sketch of spatial variation can be compared with the temporal experimental observations presented in Figure 16.

mechanism, the results are extremely promising in the region dominated solely by an approximation to the dynamic forcing required by the idealized resolvent analysis. Particularly encouraging is the extended downstream persistence of the excited mode (the low decay rate), which suggests that a significant relaxation of the actuator spacing requirements suggested by Gad-el Hak ${ }^{3}$ may be obtained by using spatially discrete forcing. Along these lines, note that the first singular value associated with this predicted mode has a magnitude $\sigma_{1}=2-5 \times 10^{3},{ }^{24}$ implying that only a tiny component (in the most amplified $\breve{\mathbf{f}}_{K}$ direction) of the relatively strong forcing input is responsible for the large (finite) amplitude of the observed velocity mode. In other words, while the coupling between the input forcing and the flow itself is important for the observed persistence of the synthetic large scale, it was not optimized by the actuation described here. Design of a more efficient coupling utilizing a subtler, less disruptive means of perturbing the flow may well be possible, subject to the requirement of overwhelming the natural internal forcing. A topic of ongoing investigation is the selection of the optimal three-dimensional wavenumber/frequency combination(s) needed to meet control objectives such as a change in the turbulent skin friction, or deterministic manipulation of the turbulent spectrum. Both of these problems come with measurement challenges associated with the identification of the correct wall shear stress, either as the objective or as an input to the friction velocity required for scaling purposes, and the distribution of energy in $K=(k, n, \omega)$ and $y$.

\section{CONCLUSIONS}

\section{A. Summary}

We have reviewed the connected analysis and observations of McKeon and Sharma, ${ }^{1}$ Jacobi and McKeon, ${ }^{24}$ and Sharma and McKeon, ${ }^{25}$ with the intent of giving a phenomenological justification for the use and success of a systems approach in understanding and controlling wall turbulence. Of particular note is the faithful connection to observed results and the potential for informed, minimum effort control. The frequency-domain interpretation, essentially a decomposition into propagating waves in the homogeneous directions, leads to a self-sustaining system in which the Navier-Stokes nonlinearity acts as a feedback mechanism to excite a set of sub-systems with highly directional amplification. The formulation can appear somewhat controversial because in important respects it is a linear analysis of a flow that is known to be nonlinear, but, as shown in Figure 3, this representation is complete in the divergence-free basis. In essence, the nonlinearity is accounted for implicitly through the assumption of the turbulent mean velocity profile and explicitly as providing a forcing at all wavenumber/frequency combinations, which is required to sustain the turbulent fluctuations.

Our discussion has focused on the (linear) resolvent operator relating the nonlinear interaction between scales and the velocity response modes at each wavenumber/frequency combination. The resolvent contains an analytical description, derived from the NSE, of the linear contents of Clauser's 
black box. To a large extent, its form determines the form of the lower-dimensional attractor guiding turbulent flow.

Much of the analytical development described here has been devoted to determining the appropriate basis for the inhomogeneous wall normal direction. In the spirit of a gain analysis, we used the singular value decomposition, which ranks input forcing distributions (right singular vectors) at a particular wavenumber/frequency combination, $K$, in terms of the amplification described by the singular values. The observed output will be a product of the amplitude of forcing and singular value summed over the singular values. At heart, the decomposition yields a basis for coherence in the wall normal direction.

The success of the first singular velocity response modes (left singular vectors) in describing a range of observed features in unperturbed high Reynolds number wall turbulence demonstrates that the resolvent can be considered low rank over the range of $K$ observed in real flows, and rank-1 in many cases, with little error. In particular, the rank-1 approximation appears capable of providing coupled insight into the existence and organization of coherent structure within the framework of the distribution of fluctuating velocities, which we believe to be a first for a NSE-based analysis.

We demonstrated successful coupling of a simple wall actuation scheme that respects the $K$ formulation of the theory to turbulent boundary layer flow, making use of the robustness property of the system to excite a single velocity response mode despite non-ideal actuation. We propose that this serves as a first step towards implementing practical closed loop control and note the success of using non-continuous forcing (in a streamwise sense) to excite persistent, slowly decaying structure in the flow, a phenomenon which may ease spatial distribution concerns associated with distributed actuation. In contrast to the usual nature of control problems in which the system must be (usually imperfectly) modeled in order to obtain a beneficial change to the output, our formulation raises the intriguing possibility of imposing a desirable mean velocity profile (thus fixing the form of the resolvents throughout the system) and determining the forcing distribution, over a range of $K$, required to sustain it. The linearity allows the linear superposition of forcing disturbances to excite multiple modes.

We believe that the resolvent formulation represents a promising approach towards the preand post-diction of the behavior of wall turbulence, for understanding the essential dynamics of a perturbed flow, effecting control, and developing low order models.

\section{B. Limitations of the approach}

We include here a brief description of the limitations of the resolvent analysis, both in its current formulation and in terms of extension to other flows.

A key limitation in the current analysis lies in the lack of information that emerges concerning relative amplitudes and phases between modes. This is a consequence of the use of unstructured forcing and the decomposition being optimized for "gain." Other choices of decomposition can give weight to relative phase information, including the sector-bounding analysis mentioned earlier, ${ }^{30,31}$ which will be more important where the critical layer mechanism is less dominant and non-normality drives the modes. This would seem to correspond to regions where Taylor's hypothesis fails. The amplitudes can be determined by establishing the requirements on self-sustaining sub-systems put forward in Eq. (15). The velocity response modes contain information analogous to the power spectral density, in that amplitude and spatial localization can be determined by the SVD.

The first singular response modes appear to capture many features of wall turbulence, but our ongoing work suggests that the strict rank-1 approximation may be insufficient to capture details of the globally most energetic Fourier modes projected out of DNS of turbulent pipe flow. These Fourier modes span a significant portion of the pipe radius, leading to a relatively large integral contribution to the turbulent kinetic energy despite a local amplitude distribution that is a fraction of that from other $K$ values. As such, the wall-normal coherence of these modes can be identified by proper orthogonal decomposition. The failure of the rank-1 approximation for this class of mode is associated with its extended radial span. A low-rank approximation (with rank greater than one but nonetheless less than ten) still appears to capture the majority of the energy content. We believe that the absence of this type of radially tall coherence in the rank-1 approximation is responsible for the 
absence of "bulges" or LSM in our analysis. Again, we associate these with modes for which phase is important.

The gain formulation of the SVD means that the results are in essence weighted to capture the most energetic streamwise velocity component for the majority of $K$ combinations. The success of the rank-1 approximation in capturing the other velocity components, and in particular the wall normal velocity, requires additional investigation. The Reynolds stress profiles associated with the first singular response show promising agreement with the attached eddy hypothesis and experimental observations, but a good approximation to this distribution will be required in order to sustain the (currently assumed) mean velocity profile.

With respect to application of our analysis to other canonical flows, the spatial inhomogeneity associated with a boundary layer also suggests the possibility of fundamentally different behavior to fully developed flows, particularly if the locally parallel approximation is not good.

Lastly, we note that the current level of sophistication in this approach does not support modeling based on coherent structure, a method that has been a focus of research aimed at finding low order models for wall turbulence motivated by the importance of structure to momentum transport, concentration of Reynolds stress, etc. Our work to date identifies the complexity of coherent structure, associated with a nonlinear diagnostic, in an otherwise linear model. In essence, a full description of all energetically active modes may be required to observe the full dynamics and evolution of coherent structure, which would probably preclude simple low order modeling.

\section{Future trends}

A manuscript such as this presents a useful vehicle for describing potential opportunities for the development of our analysis; we conclude by briefly outlining possible future developments ranging from further interrogation and modeling of the current formulation to extension to account for increasing flow complexity.

We believe that features of wall turbulence beyond those described in Sec. III are amenable to description using our analysis. For example, there appears to be a strong relationship between the wall-normal profiles of skewness and the amplitude modulation coefficient, in particular with respect to triadically consistent $K$ combinations that include the VLSM motion. ${ }^{25}$ These two measures can then be interpreted as giving information about the relative phases between velocity response modes, one of the missing pieces of our analysis to date, as identified above.

We have also noted the likely potential of the resolvent analysis to treat rough walls, or at least those with periodic geometries. Under this scenario, admitting a spatial variation of the mean flow and perhaps excitation of a range of frequencies due to local flow separation seem to be conceptually simple extensions likely to give insight into roughness-induced changes to the flow.

An important next step will be to "close the loop," in the sense of determining the amplitudes of the velocity response modes observed in (projected out of) real flows that are required to fulfill the condition of self-sustenance implied by the full NSE formulation of Figure 3. Once these amplitudes have been identified, a full description of energy transfer pathways and scaling with Reynolds number can be obtained. Extraction of energy from the mean flow, spatial transport, spectral transfer, and local triadic interactions responsible for sustaining the flow all follow from examination of the resolvent and energy gradients. The existence of self-sustaining turbulence kernels, or limited combinations of modes that are capable of self-exciting via triadic interaction, is a topic of current study. Well-known examples exist in the literature without the coherent propagating wave interpretation, but additional such solutions seem likely. ${ }^{25}$ We note also the likely connection between this kind of development and nonlinear traveling wave solutions identified for transitional pipe flow. ${ }^{7}$ Closed-loop control, given a sufficiently good model, also seems a logical next step.

As noted in Sec. V B, our analysis begins by assuming a mean velocity profile which must be sustained by the combined action of the perturbations about it. Although the resolvent associated with the mean velocity is contained in the formulation via the $K_{0}$ mode, a means of approximating it and identifying the sensitivity of the response modes to deviation from the true profile would be useful, to say the least. Towards this end, one of the authors has investigated a two-dimensional, three-component (" $2 \mathrm{D} / 3 \mathrm{C}$ ") model which appears to isolate the appropriate dominant nonlinear 
mechanism governing the mean velocity ${ }^{72,73}$ Future advances seem feasible. In this vein, we note that all the turbulent mean profiles we have encountered lead to stable $\mathcal{L}_{K}$. With appropriate tribute to Malkus, ${ }^{74}$ we conjecture that the turbulent mean velocity profile $\mathbf{u}_{0}$ always leads to stable $\mathcal{L}_{K}$, to avoid a singular resolvent response.

We have described here how a straightforward analysis permits significant simplifications in complexity, dimension, and interpretation of the NSE. The most important of these may be the low dimensionality and sparse nature of the formulation, which open the door to the use of stateof-the-art mathematical tools including compressed sampling and techniques for low rank matrix approximation of linear operators. ${ }^{31} \mathrm{~A}$ first study in a turbulent channel, ${ }^{37}$ enabled by an efficient randomized algorithm for the approximation of the SVD of low rank operators, ${ }^{75}$ gives encouraging results with respect to utilizing the low rank nature of the resolvent for both prediction and modeling purposes: it appears that a very good approximation to the distribution of streamwise turbulent intensity (the dominant contributor to the turbulent kinetic energy) can be obtained using only the first singular mode and extraordinarily simple assumptions about the amplitude distribution in $K$ space. The approximately sparse nature of the modes identified as being active in real flows in the frequency domain naturally leads to consideration of compressive sampling as a tool for data reduction, a subject of ongoing work.

Finally, we propose that the success of the resolvent analysis described here for incompressible, Newtonian flow in canonical flow configurations suggests promise for generalization to more complex flows. In particular, work is ongoing to consider adaptation of the formulation to consider rough-wall, non-Newtonian, and compressible flows, and spatial inhomogeneity. The analysis in canonical geometries is aided by the fact that there is only one inhomogeneous direction. However, we note that the requisite machinery-namely, forming the linear operator $\mathcal{L}_{K}$, shift-and-invert, and the singular value decomposition-have all been implemented in the context of global modes and transient growth in complex geometries. ${ }^{76-79}$ Therefore, the extension to flows with complex geometries should proceed similarly. This will facilitate understanding and control of a broad range of practical and applied flows.

\section{ACKNOWLEDGMENTS}

The work reported here is the result of a collaboration that began under the guidance of Professor Jonathan Morrison and Professor David Limebeer when the senior authors were postdoctoral scholars at Imperial College London, and we thank them for the insight and encouragement to work at the interface between wall turbulence and systems analysis. It is also a pleasure to acknowledge useful conversations with many giants in the field, especially Professor Ron Adrian, Professor Javier Jiménez, and Professor Peter Schmid. Other contributions to this work, not reported here, have been made by members, past and present, of the McKeon research group.

We are grateful for the support of Air Force Office of Scientific Research under Grant Nos. FA 9550-08-1-0049 and FA 9550-09-1-0701, and the National Science Foundation under Grant No. 0747672 (B.J.M.). For the early stages of this work, an Imperial College Junior Research Fellowship and the Engineering and Physical Sciences Research Council grant EP/E017304/1 are gratefully acknowledged (A.S.).

${ }^{1}$ B. J. McKeon and A. S. Sharma, "A critical layer model for turbulent pipe flow," J. Fluid Mech. 658, 336-382 (2010).

${ }^{2}$ J. Alonso, H. Balakrishnan, I. Kroo, and C. Tomlin, "Energy-efficient air transportation," in The Impact of Control Technology, edited by T. Samad and A. M. Annaswamy (IEEE Control Systems Society, 2011).

${ }^{3}$ M. Gad-el Hak, Flow Control: Passive, Active and Reactive Flow Management (Cambridge University Press, 2000).

${ }^{4}$ I. Marusic, R. Mathis, and N. Hutchins, "Predictive model for wall-bounded turbulent flow," Science 329, 193-196 (2010).

${ }^{5}$ J. Kim, "Physics and control of wall turbulence," Philos. Trans. R. Soc. London, Ser. A 369, 1396-1411 (2011).

${ }^{6}$ F. H. Clauser, "The turbulent boundary layer," Adv. Appl. Mech. 4, 1-51 (1956).

${ }^{7}$ R. R. Kerswell, "Recent progress in understanding the transition to turbulence in a pipe," Nonlinearity 18, R17 (2005).

${ }^{8}$ B. Eckhardt, T. M. Schneider, B. Hof, and J. Westerweel, "Turbulence transition in pipe flow," Annu. Rev. Fluid Mech. 39, 447-468 (2007).

${ }^{9}$ L. N. Trefethen, A. E. Trefethen, S. Reddy, and T. A. Driscoll, "Hydrodynamic stability without eigenvalues," Science 261, 578-584 (1993). 
${ }^{10}$ K. Butler and B. Farrell, "Three-dimensional optimal perturbations in viscous shear flow," Phys. Fluids A 4, 1637-1650 (1992).

${ }^{11}$ P. J. Schmid and D. S. Henningson, Stability and Transition in Shear Flows (Springer-Verlag, New York, 2001).

${ }^{12} \mathrm{~K}$. Butler and B. Farrell, "Optimal perturbations and streak spacing in wall-bounded turbulent shear flow," Phys. Fluids A 5, 774-777 (1993).

${ }^{13}$ J. C. del Álamo and J. Jiménez, "Linear energy amplification in turbulent channels," J. Fluid Mech. 559, 205-213 (2006).

${ }^{14}$ C. Cossu, G. Pujals, and S. Depardon, "Optimal transient growth and very large scale structures in turbulent boundary layers," J. Fluid Mech. 619, 79-94 (2009).

${ }^{15}$ Y. Hwang and C. Cossu, "Linear non-normal energy amplification of harmonic and stochastic forcing in the turbulent channel flow," J. Fluid Mech. 664, 51-73 (2010).

${ }^{16}$ B. Bamieh and M. Dahleh, "Energy amplification in channel flows with stochastic excitation," Phys. Fluids 13, 3258-3269 (2001).

${ }^{17}$ M. Jovanovic and B. Bamieh, "Frequency domain analysis of the linearized Navier-Stokes equations," in Proceedings of the 2003 American Control Conference, Denver, CO (IEEE, 2003), pp. 3190-3195.

${ }^{18}$ M. R. Jovanovic and B. Bamieh, "Componentwise energy amplification in channel flows," J. Fluid Mech. 534, 145-183 (2005).

${ }^{19} \mathrm{~F}$. Waleffe, "The nature of triad interactions in homogeneous turbulence," Phys. Fluids A 4, 350-363 (1992).

${ }^{20}$ J. Kim and J. Lim, "A linear process in wall-bounded turbulent shear flows," Phys. Fluids 12, 1885-1888 (2000).

${ }^{21}$ K. Zhou, J. C. Doyle, and K. Glover, Robust and Optimal Control (Prentice-Hall, New Jersey, 1996).

${ }^{22}$ W. J. Green and D. J. N. Limebeer, Linear Robust Control, edited by T. Kailath (Prentice-Hall, New Jersey, 1995).

${ }^{23}$ S. Herculano-Houzel, "The human brain in numbers: A linearly scaled-up primate brain," Front. Hum. Neurosci. 31, 1-11 (2009).

${ }^{24}$ I. Jacobi and B. J. McKeon, "Dynamic roughness-perturbation of a turbulent boundary layer," J. Fluid Mech. 688, 258-296 (2011).

${ }^{25}$ A. S. Sharma and B. J. McKeon, "On coherent structure in wall turbulence," arXiv:1301.7580.

${ }^{26}$ B. J. McKeon, "Experimental manipulation of wall turbulence," Bull. Am. Phys. Soc. 56(18), 241 (2011).

${ }^{27}$ B. J. McKeon, J. Li, W. Jiang, J. F. Morrison, and A. J. Smits, "Further observations on the mean velocity distribution in fully developed pipe flow," J. Fluid Mech. 501, 135-147 (2004).

${ }^{28}$ J. F. Morrison, W. Jiang, B. J. McKeon, and A. J. Smits, "Scaling of the streamwise velocity component in turbulent pipe flow," J. Fluid Mech. 508, 99-131 (2004).

${ }^{29}$ B. Hof, A. de Lozar, M. Avila, X. Tu, and T. M. Schneider, "Eliminating turbulence in spatially intermittent flows," Science 327, 1491-1494 (2010).

${ }^{30}$ A. S. Sharma, J. F. Morrison, B. J. McKeon, D. J. N. Limebeer, W. H. Koberg, and S. J. Sherwin, "Relaminarisation of $R e_{\tau}=100$ channel flow with globally stabilising linear feedback control," Phys. Fluids 23, 125105 (2011).

${ }^{31}$ A. S. Sharma, "Model reduction of turbulent fluid flows using the supply rate," Int. J. Bifurcation Chaos 19, 1267 (2009).

${ }^{32}$ P. J. Schmid, "Nonmodal stability theory," Annu. Rev. Fluid Mech. 39, 129-162 (2007).

${ }^{33}$ A. Meseguer and L. N. Trefethen, "Linearized pipe flow to Reynolds number 107," J. Comput. Phys. 186, 178-197 (2003).

${ }^{34}$ N. Young, An Introduction to Hilbert Space (Cambridge University Press, Cambridge, UK, 1988).

${ }^{35} \mathrm{~K}$. R. Sreenivasan, "A unified view of the origin and morphology of the turbulent boundary layer structure," in Turbulence Management and Relaminarisation; Proceedings of the IUTAM Symposium, Bangalore, India, 13-23 January 1987 (A8910154 01-34) (Springer-Verlag, Berlin/New York, 1988), pp. 37-61.

${ }^{36}$ S. A. Maslowe, "Critical layers in shear flows," Annu. Rev. Fluid Mech. 18, 405-432 (1986).

${ }^{37}$ R. Moarref, A. S. Sharma, J. A. Tropp, and B. J. McKeon, "Reynolds number scaling of the low rank approximation to turbulent channel flow," arXiv:1302.1594.

${ }^{38}$ A. J. Smits, B. J. McKeon, and I. Marusic, "High Reynolds number wall turbulence,” Annu. Rev. Fluid Mech. 43, 353-375 (2011).

${ }^{39}$ W. R. B. Morrison and R. E. Kronauer, "Structural similarity for fully developed turbulence in smooth tubes," J. Fluid Mech. 39, 117-141 (1969).

${ }^{40}$ W. R. B. Morrison, K. J. Bullock, and R. E. Kronauer, “Experimental evidence of waves in the sublayer," J. Fluid Mech. 47(4), 639-656 (1971).

${ }^{41}$ D. Dennis and T. Nickels, "On the limitations of Taylor's hypothesis in constructing long structures in wall-bounded turbulent flow," J. Fluid Mech. 614, 197-206 (2008).

42 J. LeHew, M. Guala, and B. J. McKeon, "A study of the three-dimensional spectral energy distribution in a zero pressure gradient turbulent boundary layer," Exp. Fluids 51, 997-1012 (2011).

${ }^{43}$ J. P. Monty, N. Hutchins, H. C. H. Ng, I. Marusic, and M. S. Chong, "A comparison of turbulent pipe, channel and boundary layer flows," J. Fluid Mech. 632, 431-442 (2009).

${ }^{44}$ D. Dennis and T. Nickels, "Experimental measurement of large-scale three-dimensional structures in a turbulent boundary layer. Part 1. Vortex packets," J. Fluid Mech. 673, 180-217 (2011).

${ }^{45}$ S. J. Kline, W. C. Reynolds, F. A. Schraub, and P. W. Runstadler, "The structure of turbulent boundary layers," J. Fluid Mech. 30, 741-772 (1967).

${ }^{46}$ F. Waleffe, "On a self-sustaining process in shear flows," Phys. Fluids 9, 883-900 (1997)

${ }^{47}$ J. Jiménez and A. Pinelli, "The autonomous cycle of near-wall turbulence," J. Fluid Mech. 389, 335-359 (1999).

${ }^{48}$ W. Schoppa and F. Hussain, "Coherent structure generation in near-wall turbulence," J. Fluid Mech. 453, 57-108 (2002).

${ }^{49}$ J. C. Klewicki, M. M. Metzger, E. Kelner, and E. M. Thurlow, "Viscous sublayer flow visualizations at $R_{\theta} \sim 1500000, "$ Phys. Fluids 7, 857-863 (1995).

${ }^{50}$ B. J. McKeon, A. S. Sharma, and I. Jacobi, "Predicting structural and statistical features of wall turbulence," arXiv:1012.0426. 
${ }^{51}$ N. Hutchins and I. Marusic, "Large-scale influences in near-wall turbulence," Philos. Trans. R. Soc. London, Ser. A 365, 647-664 (2007).

${ }^{52}$ D. Chung and B. J. McKeon, "Large-eddy simulation investigation of large-scale structures in a long channel flow," J. Fluid Mech. 661, 341-364 (2010).

${ }^{53}$ M. Tutkun, W. K. George, J. Delville, J.-M. Foucaut, S. Coudert, and M. Stanislas, "Space-time correlations from a 143 hot-wire rake in a high Reynolds number turbulent boundary layer," AIAA Paper 2008-4239 2008.

${ }^{54}$ M. Guala, M. J. Metzger, and B. J. McKeon, "Interactions within the turbulent boundary layer at high Reynolds number," J. Fluid Mech. 666, 573-604 (2011).

${ }^{55}$ R. Mathis, N. Hutchins, and I. Marusic, "Large-scale amplitude modulation of the small-scale structures of turbulent boundary layers," J. Fluid Mech. 628, 311-337 (2009).

${ }^{56}$ J. Jeong and F. Hussain, "On the identification of a vortex," J. Fluid Mech. 285, 69-94 (1995).

${ }^{57}$ P. Chakraborty, S. Balachandar, and R. J. Adrian, "On the relationships between local vortex identification schemes," J. Fluid Mech. 535, 189-214 (2005).

${ }^{58}$ J. Carlier and M. Stanislas, "Experimental study of eddy structures in a turbulent boundary layer using particle image velocimetry,” J. Fluid Mech. 535, 143-188 (2005).

${ }^{59}$ R. J. Adrian, C. D. Meinhart, and C. D. Tomkins, "Vortex organization in the outer region of the turbulent boundary layer," J. Fluid Mech. 422, 1-54 (2000).

${ }^{60}$ D. Dennis and T. Nickels, "Experimental measurement of large-scale three-dimensional structures in a turbulent boundary layer. Part 2. Long structures," J. Fluid Mech. 673, 218-244 (2011).

${ }^{61}$ T. Bewley, "Flow control: New challenges for a new Renaissance," Prog. Aerosp. Sci. 37, 21-58 (2001).

${ }^{62}$ L. I. Cattafesta and M. Sheplak, "Actuators for active flow control," Annu. Rev. Fluid Mech. 43, 247-272 (2011).

${ }^{63}$ B. J. McKeon, "Turbulent channel flow over model "dynamic" roughness," in IUTAM Symposium on the Physics of Wall-bounded Turbulent Flows on Rough Walls. Proceedings of the IUTAM Symposium, July 2009, edited by T. Nickels (Springer, 2010), pp. 87-92.

${ }^{64}$ I. Jacobi and B. J. McKeon, "New perspectives on the impulsive roughness perturbation of a turbulent boundary layer," J. Fluid Mech. 677, 179-203 (2011).

${ }^{65}$ G. Schubauer and H. Skramstad, "Laminar-boundary-layer oscillations and transition on a flat plate," Technical Report No. 9595 (NACA, 1943).

${ }^{66}$ A. Hussain and W. Reynolds, "The mechanics of an organized wave in turbulent shear flow," J. Fluid Mech. 41, 241-258 (1970).

${ }^{67}$ A. Hussain and W. Reynolds, "The mechanics of an organized wave in turbulent shear flow. Part 2: Experimental results," J. Fluid Mech. 54, 241-261 (1972).

${ }^{68}$ W. C. Reynolds and A. K. M. F. Hussain, "The mechanics of an organized wave in shear flow. Part 3. Theoretical models and comparisons with experiment," J. Fluid Mech. 54, 263-288 (1972).

${ }^{69}$ A. Smits, S. Young, and P. Bradshaw, "The effect of short regions of high curvature on turbulent boundary layers," J. Fluid Mech. 94, 209-242 (1979).

${ }^{70}$ P. R. Bandyopadhyay and A. K. M. F. Hussain, "The coupling between scales in shear flows," Phys. Fluids 27, 2221-2228 (1984).

${ }^{71}$ I. Jacobi, "Structure of the turbulent boundary layer under static and dynamic roughness perturbation," Ph.D. dissertation (California Institute of Technology, 2012).

${ }^{72}$ D. F. Gayme, B. J. McKeon, A. Papachristodolou, B. Bamieh, and J. C. Doyle, "Streamwise constant model of turbulence in plane Couette flow," J. Fluid Mech. 665, 99-119 (2010).

${ }^{73}$ J.-L. Bourguignon and B. J. McKeon, “A streamwise-constant model of turbulent pipe flow,” Phys. Fluids 23, 095111 (2011).

${ }^{74}$ W. V. R. Malkus, "Outline of a theory of turbulent shear flow," J. Fluid Mech. 1, 521-539 (1956).

${ }^{75}$ N. Halko, P. G. Martinsson, and J. A. Tropp, "Finding structure with randomness: Probabilistic algorithms for constructing approximate matrix decompositions," SIAM Rev. 53, 217-288 (2011).

${ }^{76}$ A. S. Sharma, N. Abdessemed, S. Sherwin, and V. Theofilis, "Optimal growth of linear perturbations in low pressure turbine flows," in IUTAM Symposium on Flow Control and MEMS, edited by J. F. Morrison (Springer, London, 2008), pp. 339-343.

${ }^{77}$ N. Abdessemed, A. S. Sharma, S. J. Sherwin, and V. Theofilis, "Transient growth analysis of the flow past a circular cylinder," Phys. Fluids 21, 044103 (2009).

${ }^{78}$ A. Sharma, N. Abdessemed, S. Sherwin, and V. Theofilis, "Transient growth mechanisms of low Reynolds number flow over a low-pressure turbine blade,” Theor. Comput. Fluid Dyn. 25, 19-30 (2011).

${ }^{79}$ V. Theofilis, "Global linear instability," Annu. Rev. Fluid Mech. 43, 319-352 (2011). 